\title{
A MORSE COMPLEX FOR LORENTZIAN GEODESICS*
}

\author{
ALBERTO ABBONDANDOLO ${ }^{\dagger}$ AND PIETRO MAJER ${ }^{\dagger}$
}

\begin{abstract}
We prove the Morse relations for the set of all geodesics connecting two non-conjugate points on a class of globally hyperbolic Lorentzian manifolds. We overcome the difficulties coming from the fact that the Morse index of every geodesic is infinite, and from the lack of the Palais-Smale condition, by using the Morse complex approach.
\end{abstract}

Key words. Geodesic, Lorentzian manifold, Morse complex.

AMS subject classifications. 58E10, 53C50

Introduction. Let $M$ be a smooth connected manifold without boundary of dimension $n+1$, and let $h$ be a Lorentzian structure on $M$ : this means that $h$ is a non-degenerate symmetric (0,2)-tensor on $M$ having $n$ positive eigenvalues and one negative eigenvalue (see [O'N83] and [BEE96] for foundational results on Lorentzian geometry). The Lorentzian structure $h$ induces a unique Levi-Civita covariant derivative $\nabla$ on $M$, and a geodesic on $(M, h)$ is a curve $\gamma: I \rightarrow M$ whose velocity $\gamma^{\prime}$ is parallelely transported with respect to this covariant derivative.

We are interested in the problem of classifying all geodesics connecting two fixed points $z_{0}, z_{1}$ in $M$. These geodesics are critical points of the energy functional

$$
E(\gamma)=\frac{1}{2} \int_{0}^{1} h\left(\gamma^{\prime}(t), \gamma^{\prime}(t)\right) d t
$$

defined on the Hilbert manifold $\mathscr{M}$ consisting of the curves $\gamma:[0,1] \rightarrow M$ connecting $z_{0}$ to $z_{1}$, of Sobolev class $W^{1,2}$.

In the Riemannian case, the geodesics $\gamma$ connecting $z_{0}$ to $z_{1}$ can be classified according to their Morse index $i(\gamma)$, that is the number of negative eigenvalues of the second differential of the Riemannian energy functional at $\gamma$. This index has also other geometrical interpretations: it coincides with $i_{\text {con }}(\gamma)$, the number of conjugate points along $\gamma$, counted with multiplicity, and with the Maslov index $i_{\text {Maslov }}(\gamma)$ of a path of Lagrangian subspaces obtained by passing to the Hamiltonian formulation and linearizing the geodesic flow along $\gamma$. When the Riemannian manifold $M$ is complete and when $z_{1}$ is not conjugated to $z_{0}$ along any geodesic, the geodesics connecting $z_{0}$ to $z_{1}$ satisfy the following Morse relations. Denote by $c_{k} \in \mathbb{N} \cup\{+\infty\}$ the number of geodesics connecting $z_{0}$ to $z_{1}$ with Morse index $k$. Then there exists a formal series $Q$ with coefficients in $\mathbb{N} \cup\{+\infty\}$ such that

$$
\sum_{k=0}^{\infty} c_{k} x^{k}=\sum_{k=0}^{\infty} \operatorname{rank} H_{k}(\Omega(M)) x^{k}+(1+x) Q(x),
$$

where $\Omega(M)$ is the space of based loops on $M$, and $H_{k}$ denotes singular homology with integer coefficients (for a modern presentation of this famous result by Morse see [Pal63]). Equivalently, the Morse relations (1) can be restated in the following way: denoting by $C_{k}$ the free Abelian group generated by the geodesic connecting $z_{0}$

\footnotetext{
*Received December 27, 2007; accepted for publication April 18, 2008.

$\dagger$ Dipartimento di Matematica, Università di Pisa, Largo Bruno Pontecorvo 5, 56127 Pisa, Italy (abbondandolo@dm.unipi.it; majer@dm.unipi.it).
} 
to $z_{1}$ with Morse index $k$, there exists homomorphisms $\partial_{k}: C_{k} \rightarrow C_{k-1}$ such that $\left\{C_{k}, \partial_{k}\right\}_{k \in \mathbb{N}}$ is a chain complex with homology isomorphic to the singular homology of $\Omega(M)$. Actually, the chain complex $\left\{C_{k}, \partial_{k}\right\}_{k \in \mathbb{N}}$ can be defined as the cellular chain complex associated to a cellular filtration of $\mathscr{M}$ produced by the negative gradient flow of the Riemannian energy functional (see for instance [Kli78] or [AM06]).

In the Lorentzian case, all the critical points of the energy functional have infinite Morse index. However, a finite relative index $i_{\text {rel }}(\gamma)$ can be defined in the following way. We fix a distribution $W$ of hyperplanes in $T M$ on which $h$ is positive definite, and we define $\mathscr{W}$ as the subbundle of $T \mathscr{M}$ such that $\mathscr{W}(\gamma)$ is the space of $W^{1,2}$ sections of $\gamma^{*}(T M)$ with values in $W$. Then the relative Morse index of a critical point $\gamma$ of $E$ on $\mathscr{M}$ is the integer

$$
i_{\text {rel }}(\gamma)=\operatorname{ind}\left(V^{-}\left(\nabla^{2} E(\gamma)\right), \mathscr{W}(\gamma)\right),
$$

where $V^{-}\left(\nabla^{2} E(\gamma)\right)$ is the negative eigenspace of the Hessian of $E$ at $\gamma$, and ind $(\mathscr{V}, \mathscr{W})$ denotes the Fredholm index of the pair $(\mathscr{V}, \mathscr{W})$, that is the integer $\operatorname{dim} \mathscr{V} \cap \mathscr{W}-$ $\operatorname{codim}(\mathscr{V}+\mathscr{W})$.

On the other hand, the Maslov index $i_{\text {Maslov }}(\gamma)$ of the geodesic $\gamma$ is still a well defined integer, which can now be also negative. Musso, Pejsachowicz, and Portaluri [MPP05] have shown that an index $i_{\operatorname{con}}(\gamma)$, counting the conjugate points along $\gamma$, can still be defined: the difficulty given by the fact that in the case of a non-positive $h$ conjugate points may accumulate can be overcome by defining $i_{\text {con }}$ as the topological degree of a map obtained by extending the equation for Jacobi fields to the complex plane. Results by these authors together with previous results by Piccione and Tausk [PT02], imply that these indices coincide ${ }^{1}$ :

$$
i_{\text {rel }}(\gamma)=i_{\text {con }}(\gamma)=i_{\text {Maslov }}(\gamma)
$$

As in the Riemannian case, this relative Morse index plays an important role in the local bifurcation theory of geodesics, see for instance [PPT04] and [PP05].

A global Morse theory for time-like geodesics (that is geodesics $\gamma$ such that $h\left(\gamma^{\prime}, \gamma^{\prime}\right)<0$ ) was developed by Uhlenbeck [Uhl75] for a class of time oriented globally hyperbolic Lorentzian manifolds: time orientability means that there exists a smooth vector field $v$ on $M$ such that $h(v, v)<0$, while global hyperbolicity requires that for every pair of points $z_{0}, z_{1}$ in $M$ the space of time-like geodesics joining them is pre-compact in the compact-open topology. Geroch has discovered in [Ger70] that a time oriented globally hyperbolic manifold $M$ is isometric to a product $X \times \mathbb{R}, X$ an $n$-dimensional manifold, with Lorentzian structure of the form

$$
h=\alpha_{i j}(x, y) d x^{i} \otimes d x^{j}-\beta(x, y) d y^{2},
$$

where $(x, y)=\left(x^{1}, \ldots, x^{n}, y\right)$ is a local coordinate system in $X \times \mathbb{R}$, the matrix $\alpha_{i j}(x, y)$ is positive definite and $\beta$ is a positive function (see [BS03] and [BS05] for a rigorous proof). Then Uhlenbeck's theory makes use of the fact that time-like geodesics locally maximize the Lorentzian length, so that methods from standard Morse theory can be applied. Uhlenbeck's results imply that the time-like geodesics joining two given non-conjugate points $z_{0}=\left(x_{0}, y_{0}\right)$ and $z_{1}=\left(x_{1}, y_{1}\right)$ satisfy Morse relations analogous to (1), where the homology of the based loop space is replaced by the homology of the space of all time-like curves joining $z_{0}$ and $z_{1}$. The homology of

\footnotetext{
${ }^{1}$ More generally, this is true for an arbitrary semi-Riemannian manifold.
} 
this space depends on the end-points $z_{0}=\left(x_{0}, y_{0}\right)$ and $z_{1}=\left(x_{1}, y_{1}\right)$, and as $y_{1}$ tends to $+\infty$ it converges to the homology of the based loop space $\Omega(X)$, so the Riemannian Morse relations are somehow recovered in the limit of two points infinitely far in time.

The aim of his paper is to show that if one considers all geodesics joining $z_{0}$ and $z_{1}$, full Morse relations hold without taking the limit for $y_{1} \rightarrow+\infty$. Here is the main result of this paper:

TheOREm. Let $M=X \times \mathbb{R}$ be a connected manifold endowed with the Lorentzian structure $h$ defined by (3). Assume that $X$ is compact and that there is some $s_{0}$ such that $X \times]-s, s\left[\right.$ is convex for every $s>s_{0}$. Let $z_{0}$ and $z_{1}$ be two points in $\left.X \times\right]-s_{0}, s_{0}[$, assumed to be non-conjugate along every geodesic joining them. Given $k \in \mathbb{Z}$, let $C_{k}$ be the free Abelian group generated by the geodesics $\gamma$ joining $z_{0}$ and $z_{1}$ of relative index $k$. Then there are boundary homomorphisms $\partial_{k}: C_{k} \rightarrow C_{k-1}$ such the homology of the chain complex $\left\{C_{k}, \partial_{k}\right\}_{k \in \mathbb{Z}}$ is isomorphic to the singular homology of $\Omega(X)$, the based loop space of $X$.

We recall that an open subset $\Omega \subset M$ is said to be convex if every geodesic $\gamma:]-\epsilon, \epsilon[\rightarrow M$ with $\gamma(0) \in \partial \Omega$ and $\gamma(]-\epsilon, \epsilon[) \subset \bar{\Omega}$ satisfies $\gamma(]-\epsilon, \epsilon[) \subset \partial \Omega$. The convexity assumption required in the above result is equivalent to asking the matrix $\left(\partial_{y} \alpha_{i j}(x, y)\right)$ to be non-positive for $s>s_{0}$ and non-negative for $s<-s_{0}$. Once $z_{0}$ is fixed, the assumption that $z_{1}$ should be non-conjugate to $z_{0}$ along any geodesic holds for a residual set of points $z_{1}$ (see [Uhl75], Theorem 1 (a)). The above theorem holds also if we replace the compactness hypothesis on $X$ by suitable bounds on the coefficients of the Lorentzian structure (see Theorem 2.1 below). Note that, although hyperbolic Lorentzian manifolds may have geodesics with negative relative index, the contribution to homology of such geodesics is null.

A Morse theory of this kind involving all geodesics connecting two non-conjugate points has been developed by Masiello (see [Mas94], Chapter 4) for a class of standard stationary Lorentzian manifolds, that is in the case of a Lorentzian structure of the form

$$
h=\alpha_{i j}(x) d x^{i} \otimes d x^{j}+\delta_{i}(x)\left(d x^{i} \otimes d y+d y \otimes d x^{i}\right)-\beta(x) d y^{2} .
$$

This is a much smaller class than the class of time oriented globally hyperbolic manifolds. Given some curve $x: I \rightarrow X$, the equation for the $y$-component of a geodesic can be uniquely solved, and solving the geodesic equation is equivalent to solving a second order ODE for $x$ associated to a nice coercive functional. As a consequence, on a standard stationary Lorentzian manifold all the geodesics have non-negative index, and the homology of $\Omega(X)$ is recovered.

Arbitrary geodesics on globally hyperbolic Lorentzian manifolds of the kind considered in the above theorem were studied by Benci, Fortunato, and Masiello in [BFM94] (see also [Mas94], Chapter 8). By using finite dimensional reductions on the $y$-component of the geodesic equation, they proved the existence of at least one geodesic connecting $z_{0}$ to $z_{1}$. Actually, their proof could be refined to prove the weak Morse relations, that is the inequalities $c_{k} \geq \operatorname{rank} H_{k}(\Omega(X))$ for every $k \in \mathbb{N}$. See also the review paper [ABFM03].

The strong Morse relations stated in our main theorem seem to go beyond the methods used in [BFM94]. One of the main difficulties in this problem is that the energy functional of a globally hyperbolic Lorentzian manifold may fail to satisfy the Palais-Smale condition at every energy level (unlike the stationary case). This 
problem is overcome in [BFM94] by proving an a priori estimate on the $C^{1}$ norm of the second component of any curve $\gamma=(x, y)$ solving $\nabla E(x, y)=\lambda y$ for some $\lambda \geq 0$, with energy $E(\gamma)$ bounded above. Here we need to sharpen this estimate, by proving that

$$
\nabla E(x, y)=\lambda y \text { with } \lambda \geq 0 \quad \Rightarrow \quad\|y\|_{C^{1}}^{2} \leq p \max \{E(x, y), 0\}+q,
$$

for suitable constants $p, q$ (see Lemma 3.1 below) ${ }^{2}$. Here is where the convexity assumption is used.

Then our argument uses in an essential way the Morse complex approach developed in [AM05, AM08], instead than classical Morse theory applied to finite dimensional reductions.

The idea is to define the boundary homomorphism $\partial_{k}: C_{k} \rightarrow C_{k-1}$ by counting the negative gradient flow lines of $E$ connecting critical points of relative Morse index difference one ${ }^{3}$. The a priori estimate (4) allows to build a pseudo-gradient vector field for $E$ such that all the flow lines connecting two critical points are bounded. Since the Palais-Smale condition holds on bounded subsets of $\mathscr{M}$, one gets enough compactness to define the boundary operator $\partial_{k}$. Here weaker a priori estimates as the one proved in [BFM94] would be sufficient, but the estimate (4) is used in an essential way in the computation of the homology of the resulting chain complex. Indeed, estimate (4) allows us to prove that this homology is stable with respect to $C^{0}$ perturbations of the Lorentzian metric. Then the conclusion follows from a connection argument, together with the fact that in the particular case of a Lorentzian metric of the form $h=\alpha_{i j}(x) d x^{i} \otimes d x^{j}-d y^{2}$ the resulting homology is just the homology of the space of based loops on $X$, by the Morse complex reinterpretation of classical infinite dimensional Morse theory.

1. The Morse complex: an abstract setting. In this section we give a brief account of the construction of the Morse complex for a class of gradient-like flows on an infinite dimensional manifold. The general theory - which holds for a much larger class - is fully described in [AM05] and [AM08].

Let $\mathscr{X}$ be a Hilbert manifold, endowed with a complete Riemannian structure, let $\mathscr{Y}$ be an affine Hilbert space modeled on the Hilbert space $H$, and let $\mathscr{M}=\mathscr{X} \times \mathscr{Y}$ be the product manifold, endowed with the product Riemannian structure. We denote by $P: T \mathscr{M} \rightarrow H$ the map

$$
(x, y, \xi, \eta) \mapsto \eta, \quad x \in \mathscr{X}, y \in \mathscr{Y}, \xi \in T_{x} \mathscr{X}, \eta \in T_{y} \mathscr{Y}=H,
$$

that is the differential of the projection of $\mathscr{X} \times \mathscr{Y}$ onto the second factor.

The set of zeroes of a vector field $F$ on $\mathscr{M}$ is denoted by rest $(F)$. A smooth vector field $F$ on $\mathscr{M}$ is said to be a Morse vector field if for every $z \in$ rest $(F)$ the Jacobian of $F$ at $z$, denoted by $\nabla F(z)$, is an infinitesimally hyperbolic operator on $T_{z} \mathscr{M}$ (i.e. its spectrum is disjoint from the imaginary axis $i \mathbb{R})$. The positive and the negative eigenspaces of $\nabla F(z)$ are denoted by $V^{+}(\nabla F(z))$ and $V^{-}(\nabla F(z))$, respectively.

The set of critical points of a smooth function $f: \mathscr{M} \rightarrow \mathbb{R}$ is denoted by $\operatorname{crit}(f)$. A smooth function $f: \mathscr{M} \rightarrow \mathbb{R}$ is said to be a Lyapunov function for $F$ if $D f(p)[F(p)]<0$

\footnotetext{
${ }^{2}$ Actually, in [BFM94] the convexity assumption is replaced by a sort of convexity at infinity. This is a weaker assumption which does not imply (4)

${ }^{3}$ This is the basic idea of Floer homology, but in a different contest. Here indeed we are dealing with a well-defined gradient flow on an infinite dimensional Hilbert manifold, whereas Floer homology deals with an elliptic PDE (see [Flo89]).
} 
for every $p$ in $\mathscr{M}$ which is not a rest point of $F$. If moreover for every critical point $z$ the Hessian $\nabla^{2} f(z)$ is an isomorphism (i.e. $f$ is a Morse function), and $-\nabla^{2} f(z)$ is strictly positive (resp. strictly negative) on $V^{+}(\nabla F(z))$ (resp. on $\left.V^{-}(\nabla F(z))\right)$, $f$ is said to be a non-degenerate Lyapunov function for $F$. In this case, rest $(F)=\operatorname{crit}(f)$, and $F$ is said a (negative) pseudo-gradient for $f$.

A Palais-Smale sequence for the pair $(F, f)$ is a sequence $\left(p_{n}\right) \subset \mathscr{M}$ such that $f\left(p_{n}\right)$ is bounded and $D f\left(p_{n}\right)\left[F\left(p_{n}\right)\right]$ is infinitesimal.

Let $\mathscr{A}$ be an open subset of $\mathscr{M}$, and let $a: \mathscr{M} \rightarrow \mathbb{R}$ be a smooth function such that $\mathscr{A}=\{a<0\}$. Let $F$ be a smooth Morse vector field on $\mathscr{M}$, and let $f$ be a smooth non-degenerate Lyapunov function for $F$. We assume the following conditions:

(F1) $F$ is bounded on $\mathscr{M}$, and Lipschitz continuous on bounded subsets of $\mathscr{M}$.

(F2) $F$ has no rest points on the closure of $\mathscr{A}$, and $a$ is a Lyapunov function for $F$ on $\mathscr{A}$.

(F3) $f$ and $a$ are bounded on bounded subsets of $\mathscr{M}$.

(F4) For every $c, c^{\prime} \in \mathbb{R}$ the subset $\{f \leq c\} \cap\left\{a \geq c^{\prime}\right\}$ is bounded.

(F5) Every bounded Palais-Smale sequence for $(F, f)$ has a converging subsequence.

(F6) For every $z=(x, y) \in \operatorname{rest}(F)$, the subspaces $V^{-}(\nabla F(z))$ and $V^{+}(\nabla F(z))$ are compact perturbations ${ }^{4}$ of $T_{x} \mathscr{M} \times(0)$ and $(0) \times T_{y} \mathscr{Y}=(0) \times H$, respectively.

(F7) For every $y \in \mathscr{Y}$, the map $\mathscr{X} \rightarrow H, x \mapsto P F(x, y)$, is compact (i.e. it maps bounded sets into pre-compact sets).

We denote by $\phi^{F}: \mathbb{R} \times \mathscr{M} \rightarrow \mathscr{M}$ the flow of $F$ (which is globally defined because $F$ is bounded and $\mathscr{M}$ is complete). Condition (F2) implies that $\mathscr{A}$ is positively invariant for $\phi^{F}$. Conditions (F3) and (F4) imply that $f$ is bounded below on $\mathscr{M} \backslash \mathscr{A}$, so by $(\mathrm{F} 2)$ and (F5) $F$ has finitely many rest points in $\{f \leq c\}$, for every $c \in \mathbb{R}$.

By (F6), for every rest point $z=(x, y)$ the pair of subspaces $\left(V^{+}(\nabla F(z)), T_{x} \mathscr{X} \times\right.$ $(0))$ is a Fredholm pair ${ }^{5}$ in $T_{z} \mathscr{M}$, and we define the relative Morse index of $z$ as the Fredholm index of such a pair,

$$
i_{\mathrm{rel}}(z):=\operatorname{ind}\left(V^{+}(\nabla F(z)), T_{x} \mathscr{X} \times(0)\right) .
$$

The stable and unstable manifolds of a rest point $z$, that is the sets

$$
\begin{aligned}
& W^{s}(z ; F):=\left\{p \in \mathscr{M} \mid \lim _{t \rightarrow+\infty} \phi^{F}(t, p)=z\right\}, \\
& W^{u}(z ; F):=\left\{p \in \mathscr{M} \mid \lim _{t \rightarrow-\infty} \phi^{F}(t, p)=z\right\},
\end{aligned}
$$

are smoothly embedded submanifolds of $\mathscr{M}$, diffeomorphic to the Hilbert spaces $V^{-}(\nabla F(z))$ and $V^{+}(\nabla F(z))$, respectively (because the Morse vector field $F$ has a Lyapunov function). Up to a perturbation, we may also assume that the vector field $F$ has the Morse-Smale property, meaning that the stable and unstable manifolds of any two rest points intersect transversally. In this situation, $W^{u}(z ; F) \cap W^{s}\left(z^{\prime} ; F\right)$ - if

\footnotetext{
${ }^{4}$ A closed linear subspace $V$ of a Hilbert space is said to be a compact perturbation of a closed linear subspace $W$ if the difference of their orthogonal projectors is a compact operator.

${ }^{5}$ A pair of closed subspaces $(V, W)$ of a Hilbert space $H$ is said to be a Fredholm pair if $V \cap W$ has finite dimension, and $V+W$ is closed and has finite codimension. The Fredholm index of $(V, W)$ is the integer ind $(V, W)=\operatorname{dim} V \cap W-\operatorname{dim} H /(V+W)$.
} 
non-empty - is a manifold of dimension $i_{\text {rel }}(z)-i_{\text {rel }}\left(z^{\prime}\right)$. An orientation of the determinant bundle ${ }^{6}$ over the Fredholm pair $\left(T_{z} W^{s}(z ; F),(0) \times H\right)$, for every $z \in \operatorname{rest}(F)$, induces an orientation of each intersection $W^{u}(z ; F) \cap W^{s}\left(z^{\prime} ; F\right)$.

Every intersection $W^{u}(z ; F) \cap W^{s}\left(z^{\prime} ; F\right)$ is pre-compact. Indeed, by (F2) this intersection is contained in $\{a \geq 0\}$, and since it is also contained in $\{f \leq f(z)\}$, it is bounded by (F4). Then (F5), (F6), and (F7) imply that such an intersection has compact closure in $\mathscr{M}$. Compactness and transversality imply that, when $i_{\text {rel }}(z)=$ $i_{\text {rel }}\left(z^{\prime}\right)+1, W^{u}(z ; F) \cap W^{s}\left(z^{\prime} ; F\right)$ consists of finitely many flow orbits. In this case, we can define an integer $d_{F}\left(z, z^{\prime}\right)$ by counting each orbit as +1 if its orientation agrees with the flow direction, as -1 otherwise. Let $C_{k}(F)$ be the free Abelian group generated by the rest points of $F$ of relative Morse index $k$, and let $\partial_{k}: C_{k}(F) \rightarrow$ $C_{k-1}(F)$ be the homomorphism defined by

$$
\partial_{k} z=\sum_{\substack{z^{\prime} \in \operatorname{rest}(F) \\ i_{\mathrm{rel}}\left(z^{\prime}\right)=k-1}} d_{F}\left(z, z^{\prime}\right) z^{\prime},
$$

for every $z \in \operatorname{rest}(F)$ with $i_{\text {rel }}(z)=k$. The above sum is finite because $F$ has finitely many rest points in $\{f \leq f(z)\}$. These homomorphisms satisfy $\partial_{k} \circ \partial_{k+1}=0$, so

$$
\left\{C_{k}(F), \partial_{k}\right\}_{k \in \mathbb{Z}}
$$

is a chain complex of Abelian groups, called the Morse complex of $F$. Changing some of the orientations of the determinant lines over $\left(T_{z} W^{s}(z ; F),(0) \times H\right)$, for $z \in \operatorname{rest}(F)$, produces an isomorphic chain complex. Changing the vector field $F$ while keeping the same Lyapunov function $f$ - hence the same rest points and the same groups $C_{k}$ - produces an isomorphic chain complex (the isomorphisms here being non-trivial). Therefore, the homology of the Morse complex depends only on the Lyapunov function $f$, and it is called the Morse homology of $f$,

$$
H_{k}(f):=H_{k}\left(\left\{C_{*}(F), \partial_{*}\right\}\right)=\operatorname{ker} \partial_{k} / \operatorname{Im} \partial_{k+1} .
$$

Remark 1.1. Assume that the data $F, f, \mathscr{A}$, a satisfy (F1)-(F7), and that the function $f$ and the vector field $F$ have the form

$$
f(x, y)=f^{+}(x)-f^{-}(y), \quad F(x, y)=F^{+}(x) \oplus\left(-F^{-}(y)\right) .
$$

where $f^{-}, f^{+}$are bounded below, and $f^{-}$has finitely many critical points. By (F6), all the critical points of $f^{+}$and $f^{-}$have finite Morse index, and $i_{\mathrm{rel}}(x, y)=i(x)-i(y)$, $i$ denoting the usual Morse index. In this case it is easily seen that

$$
C_{k}(F)=\bigoplus_{p-q=k} C_{p}\left(F^{+}\right) \otimes C_{q}\left(F^{-}\right),
$$

and that the Morse homology of $f$ is

$$
H_{k}(f)=\bigoplus_{p-q=k} H_{p}\left(f^{+}\right) \otimes H_{q}\left(f^{-}\right) .
$$

\footnotetext{
${ }^{6}$ The determinant bundle over the space of all Fredholm pairs of a Hilbert space is a real line bundle whose fiber at $(V, W)$ is $\Lambda^{\text {top }}(V \cap W) \otimes\left(\Lambda^{\text {top }}(H /(V+W))\right)^{*}$, where $\Lambda^{\text {top }}(X)$ is the degree- $n$ component of the exterior algebra of the $n$-dimensional vector space $X$.
} 
The Morse homologies of $f^{+}$and $f^{-}$are isomorphic to the singular homologies of $\mathscr{X}$ and $\mathscr{Y}$, respectively (this holds in general for a Morse function bounded from below, satisfying the Palais-Smale condition, and having critical points with finite Morse index, see [AM06]). Since $\mathscr{Y}$ has the homology of a point, we conclude that $H_{k}(f)$ is isomorphic to $H_{k}(\mathscr{X})$.

We conclude this section by describing the functorial properties of the Morse complex. Assume that $\left(F_{0}, f_{0}, \mathscr{A}_{0}, a_{0}\right)$ and $\left(F_{1}, f_{1}, \mathscr{A}_{1}, a_{1}\right)$ satisfy conditions (F1)(F7) on the same Hilbert manifold $\mathscr{M}$. Furthermore, assume the following condition:

(F8) For every $c, c^{\prime} \in \mathbb{R}$ the subset $\left\{f_{0} \leq c\right\} \cap\left\{a_{1} \geq c^{\prime}\right\}$ is bounded.

This condition implies that for every $z_{0} \in$ rest $\left(F_{0}\right)$ and $z_{1} \in \operatorname{rest}\left(F_{1}\right)$ the intersection

$$
W^{u}\left(z_{0} ; F_{0}\right) \cap W^{s}\left(z_{1} ; F_{1}\right)
$$

is bounded. Indeed, by (F2) this intersection is contained in $\left\{a_{1} \geq 0\right\}$, and being contained also in $\left\{f_{0} \leq f_{0}\left(z_{0}\right)\right\}$, it is bounded by (F8). By (F3) and (F4), also its positive evolution by $\phi^{F_{1}}$ and its negative evolution by $\phi^{F_{0}}$ are bounded. Then (F5), (F6), and (F7) imply that this intersection is pre-compact. Up to perturbing the vector fields $F_{0}$ and $F_{1}$ without affecting their Morse complexes, we may assume that the above intersections are also transverse. Then (F6) and (F7) imply that $W^{u}\left(z_{0} ; F_{0}\right) \cap W^{s}\left(z_{1} ; F_{1}\right)$ is an oriented submanifold of dimension

$$
\operatorname{dim} W^{u}\left(z_{0} ; F_{0}\right) \cap W^{s}\left(z_{1} ; F_{1}\right)=i_{\text {rel }}\left(z_{0} ; F_{0}\right)-i_{\text {rel }}\left(z_{1} ; F_{1}\right) .
$$

In particular, when $i_{\text {rel }}\left(z_{0} ; F_{0}\right)=i_{\text {rel }}\left(z_{1} ; F_{1}\right)$ this intersection is a finite set of points, each of which carries an orientation sign +1 or -1 . The sum of these numbers defines an integer $d_{F_{0}, F_{1}}\left(z_{0}, z_{1}\right)$, and the homomorphism defined by

$$
\Phi_{F_{0}, F_{1}}: C_{k}\left(F_{0}\right) \rightarrow C_{k}\left(F_{1}\right), \quad \Phi_{F_{0}, F_{1}} z_{0}=\sum_{\substack{z_{1} \in \operatorname{rest}\left(F_{1}\right) \\ i_{\text {rel }}\left(z_{1} ; F_{1}\right)=k}} d_{F_{0}, F_{1}}\left(z_{0}, z_{1}\right) z_{1},
$$

for every $z_{0} \in \operatorname{rest}\left(F_{0}\right), i_{\text {rel }}\left(z_{0} ; F_{0}\right)=k$, is a chain map from the Morse complex of $F_{0}$ to the Morse complex of $F_{1}$. Changing the vector fields $F_{0}$ and $F_{1}$ while keeping the same Lyapunov functions $f_{0}$ and $f_{1}$, produces homotopic chain maps, therefore the induced homomorphism between the Morse homology groups

$$
\Phi_{f_{0}, f_{1}}: H_{k}\left(f_{0}\right) \rightarrow H_{k}\left(f_{1}\right)
$$

is well-defined. If $f_{0}=f_{1}, \Phi_{f_{0}, f_{1}}$ is the identity.

Finally, assume that $\left(F_{0}, f_{0}, \mathscr{A}_{0}, a_{0}\right),\left(F_{1}, f_{1}, \mathscr{A}_{1}, a_{1}\right)$, and $\left(F_{2}, f_{2}, \mathscr{A}_{2}, a_{2}\right)$, satisfy (F1)-(F7), and that the pairs $\left(f_{0}, a_{1}\right)$ and $\left(f_{1}, a_{2}\right)$ satisfy (F8). Assume also:

(F9) There holds $a_{1} \leq f_{1}$, and for every $c, c^{\prime} \in \mathbb{R}$ the subsets $\left\{f_{0} \leq c\right\} \cap\left\{f_{1}+a_{1} \geq\right.$ $\left.c^{\prime}\right\}$ and $\left\{f_{1}+a_{1} \leq c\right\} \cap\left\{a_{2} \geq c^{\prime}\right\}$ are bounded.

Since

$$
\left\{f_{0} \leq c\right\} \cap\left\{a_{2} \geq c^{\prime}\right\} \subset\left(\left\{f_{0} \leq c\right\} \cap\left\{f_{1}+a_{1} \geq 0\right\}\right) \cup\left(\left\{f_{1}+a_{1} \leq 0\right\} \cap\left\{a_{2} \geq c^{\prime}\right\}\right),
$$

(F9) implies that also the pair $\left(f_{0}, a_{2}\right)$ satisfies (F8). Given $z_{0} \in \operatorname{rest}\left(F_{0}\right)$ and $z_{2} \in$ rest $\left(F_{2}\right)$, we consider the set

$$
W\left(z_{0}, z_{2}\right)=\left\{\left(z, z^{\prime}\right) \in W^{u}\left(z_{0} ; F_{0}\right) \times W^{s}\left(z_{2} ; F_{2}\right) \mid \exists t \geq 0 \text { such that } \phi^{F_{1}}(t, z)=z^{\prime}\right\} .
$$


Denote by $W_{1}$ and $W_{2}$ the projections of $W\left(z_{0}, z_{2}\right)$ onto the first and the second factor of $\mathscr{M} \times \mathscr{M}$. We claim that $f_{1}+a_{1}$ is bounded below on $W_{1}$. If not, there exists a sequence $\left(w_{n}, w_{n}^{\prime}\right) \subset W\left(z_{0}, z_{2}\right)$ such that $f_{1}\left(w_{n}\right)+a_{1}\left(w_{n}\right) \rightarrow-\infty$. Since $a_{1} \leq f_{1}$, also $a_{1}\left(w_{n}\right) \rightarrow-\infty$, so $w_{1}$ eventually belongs to $\mathscr{A}_{1}$. On this set both $f_{1}$ and $a_{1}$ are Lyapunov functions for $F_{1}$, so also their sum is a Lyapunov function for $F_{1}$, and we deduce that $f_{1}\left(w_{n}^{\prime}\right)+a_{1}\left(w_{n}^{\prime}\right) \rightarrow-\infty$. In particular, $f_{1}\left(w_{n}^{\prime}\right)+a_{1}\left(w_{n}^{\prime}\right)$ is bounded above. By condition (F2) for $F_{2}, a_{2}\left(w_{n}^{\prime}\right) \geq 0$, so (F9) implies that $\left(w_{n}^{\prime}\right)$ is bounded. But then $f_{1}\left(w_{n}^{\prime}\right)+a_{1}\left(w_{n}^{\prime}\right)$ is bounded (condition (F3)), contradicting the fact that this sequence tends to $-\infty$. This proves the claim.

This fact, together with the fact that $f_{0}$ is bounded above on $W_{1}$, implies by (F9) that $W_{1}$ is bounded. Then $f_{1}$ is bounded on $W_{1}$ (condition (F3)), hence it is bounded above on $W_{2}$. Since $a_{2}$ is bounded below on $W_{2}$, condition (F8) for the pair $\left(f_{1}, a_{2}\right)$ implies that also $W_{2}$ is bounded. By (F3) and (F4), also the negative evolution of $W_{1}$ by $\phi^{F_{0}}$, the positive evolution of $W_{2}$ by $\phi^{F_{2}}$, and the set of orbits of $\phi^{F_{1}}$ connecting $W_{1}$ to $W_{2}$ are bounded.

As before, conditions (F5), (F6), and (F7), imply that $W\left(z_{0}, z_{2}\right)$ has compact closure in $\mathscr{M} \times \mathscr{M}$, and up to perturbations, it is an oriented submanifold of dimension

$$
\operatorname{dim} W\left(z_{0}, z_{2}\right)=i_{\text {rel }}\left(z_{0} ; F_{0}\right)-i_{\text {rel }}\left(z_{2} ; F_{2}\right)+1 .
$$

When $i_{\text {rel }}\left(z_{0} ; F_{0}\right)=i_{\text {rel }}\left(z_{2} ; F_{2}\right)-1, W\left(z_{0}, z_{2}\right)$ is a finite set of points with orientation signs, and their sum defines an integer $d_{F_{0}, F_{1}, F_{2}}\left(z_{0}, z_{2}\right)$. The homomorphism

$$
\Psi: C_{k}\left(F_{0}\right) \rightarrow C_{k+1}\left(F_{2}\right), \quad \Psi z_{0}=\sum_{\substack{z_{2} \in \operatorname{rest}\left(F_{2}\right) \\ i_{\text {rel }}\left(z_{2} ; F_{2}\right)=k+1}} d_{F_{0}, F_{1}, F_{2}}\left(z_{0}, z_{2}\right) z_{2},
$$

is a chain homotopy between $\Phi_{F_{0}, F_{2}}$ and $\Phi_{F_{1}, F_{2}} \circ \Phi_{F_{0}, F_{1}}$. Therefore,

$$
\Phi_{f_{1}, f_{2}} \circ \Phi_{f_{0}, f_{1}}=\Phi_{f_{0}, f_{2}}: H_{k}\left(f_{0}\right) \rightarrow H_{k}\left(f_{2}\right),
$$

for every $k \in \mathbb{Z}$.

REMARK 1.2. Condition (F9) can be replaced by the more symmetric assumption that the set $\left\{a_{1} \leq c\right\} \cap\left\{a_{2} \geq c^{\prime}\right\}$ should be bounded for every $c, c^{\prime} \in \mathbb{R}$. For the purposes of this paper however (F9) is easier to check.

2. The main result. Let $(X, g)$ be a smooth connected complete Riemannian manifold, and let $M=X \times \mathbb{R}$ be endowed with the Lorentzian structure

$$
h(x, y)\left[\left(\xi_{1}, \eta_{1}\right),\left(\xi_{2}, \eta_{2}\right)\right]=g(x)\left[\alpha(x, y) \xi_{1}, \xi_{2}\right]-\beta(x, y) \eta_{1} \eta_{2},
$$

where $(x, y) \in X \times \mathbb{R}, \xi_{1}, \xi_{2} \in T_{x} X, \eta_{1}, \eta_{2} \in T_{y} \mathbb{R}=\mathbb{R}, \alpha$ is a smooth section of the bundle over $X \times \mathbb{R}$ of $g$-symmetric and $g$-positive $(1,1)$ tensors on $X$, and $\beta$ is a smooth positive real function on $M$. In order to emphasize the role of $\alpha$ and $\beta$, the Lorentzian energy functional is denoted by

$$
E_{\alpha, \beta}(x, y)=\frac{1}{2} \int_{0}^{1} g(x)\left[\alpha(x, y) x^{\prime}, x^{\prime}\right] d t-\frac{1}{2} \int_{0}^{1} \beta(x, y)\left|y^{\prime}\right|^{2} d t,
$$

for a curve $(x, y):[0,1] \rightarrow M$. The aim of this paper is to prove the following:

TheOREm 2.1. Let $(X, g)$ be a smooth connected complete Riemannian manifold, and let $M=X \times \mathbb{R}$ be endowed with the Lorentzian structure $h$ defined as above. Assume that: 
(a0) there is a positive number $s_{0}$ such that $\left.X \times\right]-s, s\left[\right.$ is convex for every $s>s_{0}$;

(a1) there are positive numbers $\underline{\alpha}, \bar{\alpha}$ such that $\underline{\alpha} I \leq \alpha \leq \bar{\alpha} I$ as $g(x)$-symmetric $(1,1)$ tensors, on $X \times\left[-s_{0}-1, s_{0}+1\right]$;

(a2) there are positive numbers $\beta, \bar{\beta}$ such that $\beta \leq \beta \leq \bar{\beta}$ on $X \times\left[-s_{0}-1, s_{0}+1\right]$;

(a3) the function $\xi \mapsto g\left(\partial_{y} \alpha \xi, \xi\right) / g(\alpha \xi, \xi)$ is bounded on $(T X \backslash\{0\}) \times\left[-s_{0}-1, s_{0}+\right.$ 1];

(a4) the function $\partial_{y} \beta / \beta$ is bounded on $X \times\left[-s_{0}-1, s_{0}+1\right]$.

Let $z_{0}=\left(x_{0}, y_{0}\right)$ and $z_{1}=\left(x_{1}, y_{1}\right)$ be two points in $\left.X \times\right]-s_{0}, s_{0}[$, assumed to be nonconjugate along every geodesic joining them. Given $k \in \mathbb{Z}$, let $C_{k}$ be the free Abelian group generated by the geodesics $\gamma$ joining $z_{0}$ and $z_{1}$ of relative Morse index $k$. Then there is a pseudo-gradient vector field $F$ for $E_{\alpha, \beta}$ on the Hilbert manifold of $W^{1,2}$ curves joining $z_{0}$ to $z_{1}$ such that $C_{k}(F)=C_{k}$, and the homology of the corresponding Morse complex $\left\{C_{k}(F), \partial_{k}\right\}_{k \in \mathbb{Z}}$ is isomorphic to the singular homology of $\Omega(X)$, the based loop space of $X$.

Notice that assumptions (a1)-(a4) always hold if $X$ is compact, so Theorem 2.1 implies the theorem stated in the introduction. By condition (a0), all the geodesics joining $z_{0}$ and $z_{1}$ are contained in $X \times\left[-s_{0}, s_{0}\right]$.

If $\gamma=(x, y):[0,1] \rightarrow M$ is a geodesic, the Euler-Lagrange equation for $E_{\alpha, \beta}$ with respect to variations on the second component of $(x, y)$ is the equation

$$
\left(\beta(x, y) y^{\prime}\right)^{\prime}+\frac{1}{2} g(x)\left[\partial_{y} \alpha(x, y) x^{\prime}, x^{\prime}\right]-\frac{1}{2} \partial_{y} \beta(x, y)\left|y^{\prime}\right|^{2}=0 .
$$

Moreover, the conservation of energy produces the identity

$$
\frac{1}{2} g(x(t))\left[\alpha(x(t), y(t)) x^{\prime}(t), x^{\prime}(t)\right]-\frac{1}{2} \beta(x(t), y(t))\left|y^{\prime}(t)\right|^{2}=E_{\alpha, \beta}(x, y), \quad \forall t \in[0,1] .
$$

The convexity assumption $(\mathrm{a} 0)$ can be restated in terms of the derivatives of the Lorentzian structure:

LEMma 2.2. The sets $X \times]-s, s\left[\right.$ are convex for every $s>s_{0}$ if and only if for every $x \in X$

$$
\partial_{y} \alpha(x, y) \leq 0 \quad \forall y>s_{0}, \quad \partial_{y} \alpha(x, y) \geq 0 \quad \forall y<-s_{0},
$$

as $g(x)$-symmetric $(1,1)$ tensors.

Proof. First notice that $X \times]-s, s[$ is convex if and only if every geodesic $\gamma=$ $(x, y):]-\epsilon, \epsilon[\rightarrow X \times \mathbb{R}$ with $|y(0)|=s$ and $|y(t)| \leq s$ for every $t$ satisfies $|y(t)|=s$ for every $t$.

Assume that $\partial_{y} \alpha(x, y) \leq 0$ does not hold at some point $\left(x_{0}, y_{0}\right) \in X \times \mathbb{R}$, that is there is some $\xi \in T_{x_{0}} X$ such that

$$
g\left(x_{0}\right)\left[\partial_{y} \alpha\left(x_{0}, y_{0}\right) \xi, \xi\right]>0 .
$$

Let $(x, y):]-\epsilon, \epsilon\left[\rightarrow X \times \mathbb{R}\right.$ be the geodesic such that $(x(0), y(0))=\left(x_{0}, y_{0}\right)$ and $\left(x^{\prime}(0), y^{\prime}(0)\right)=(\xi, 0)$. The geodesic equation (6) for $y$ at $t=0$ yields

$$
\beta\left(x_{0}, y_{0}\right) y^{\prime \prime}(0)+\frac{1}{2} g\left(x_{0}\right)\left[\partial_{y} \alpha\left(x_{0}, y_{0}\right) \xi, \xi\right]=0
$$

Therefore $y^{\prime \prime}(0)<0$, hence $y_{0}$ is a strict local maximum of $y$, and the set $\left.X \times\right]-\infty, y_{0}[$ is not convex. So, if $X \times]-s, s\left[\right.$ is convex then $\partial_{y} \alpha(x, s) \leq 0$ for every $x \in X$. Similarly, $\partial_{y} \alpha(x,-s) \geq 0$ for every $x \in X$. 
To prove the converse, assume (8) and choose some $s>s_{0}$. Let $(x, y)$ be a geodesic in $X \times \mathbb{R}$ with $y(0)=s$ and $y(t) \leq s$ for every $t$ (the case $y(0)=-s$ and $y(t) \geq-s$ for every $t$ being analogous). There is an interval $I$, neighborhood of 0 , on which $g(x)\left[\partial_{y} \alpha(x, y) x^{\prime}, x^{\prime}\right] \leq 0$. From the geodesic equation (6) we deduce

$$
y^{\prime \prime}(t)+u(t) y^{\prime}(t) \geq 0, \quad \forall y \in I,
$$

for a suitable smooth function $u$. Since $y(t) \leq y(0)=s$ for every $t \in I$, the strong maximum principle implies that $y(t)=s$ for every $t \in I$. This shows that $X \times]-s, s[$ is convex.

Up to changing the Lorentzian structure outside $X \times\left[-s_{0}, s_{0}\right]$ without affecting the convexity assumption (a0), we may assume that the bounds listed in (a1)-(a4) hold on the whole $X \times \mathbb{R}$. Indeed, it is enough to choose a smooth function $\psi: \mathbb{R} \rightarrow \mathbb{R}$ such that $\psi=1$ on $\left[-s_{0}, s_{0}\right], \psi=0$ on $\mathbb{R} \backslash\left[-s_{0}-1, s_{0}+1\right], \psi^{\prime} \geq 0$ on $\left[-s_{0}-1,-s_{0}\right]$, $\psi^{\prime} \leq 0$ on $\left[s_{0}, s_{0}+1\right]$, and to replace $\alpha$ and $\beta$ by

$$
\psi(y) \alpha(x, y)+(1-\psi(y)) \underline{\alpha} I, \quad \text { and } \quad \psi(y) \beta(x, y)+(1-\psi(y)) \underline{\beta} .
$$

Conditions (a1)-(a4) now hold on $X \times \mathbb{R}$. The derivative with respect to $y$ of the second function is

$$
\psi \partial_{y} \alpha+\psi^{\prime}(\alpha-\bar{\alpha})
$$

so the new Lorentzian structure satisfies also (a0), by Lemma 2.2.

By the above considerations, we may assume that the coefficients of our Lorentzian structure belong to the set $\Gamma=\Gamma\left(s_{0}, \underline{\alpha}, \bar{\alpha}, \underline{\beta}, \bar{\beta}, a, b\right)$ consisting of all $(\alpha, \beta)$ satisfying:

(h0) $\partial_{y} \alpha(x, y) \leq 0$ for every $y \geq s_{0}, \partial_{y} \alpha(x, y) \geq 0$ for every $y \leq-s_{0}$, for every $x \in X$

(h1) $\underline{\alpha} I \leq \alpha \leq \bar{\alpha} I$ on $M$;

(h2) $\underline{\beta} \leq \beta \leq \bar{\beta}$ on $M$

(h3) $\sup _{\xi \in T X \backslash\{0\}} \frac{\left|g\left(\partial_{y} \alpha \xi, \xi\right)\right|}{g(\alpha \xi, \xi)} \leq a$ on $M$;

(h4) $\left|\partial_{y} \beta\right| / \beta \leq b$ on $M$.

3. A priori bounds. The following a priori estimate sharpens Theorem 4.1 in [BFM94]:

Lemma 3.1. Let $(\alpha, \beta) \in \Gamma\left(s_{0}, \underline{\alpha}, \bar{\alpha}, \underline{\beta}, \bar{\beta}, a, b\right)$ and let $\left.y_{0}, y_{1} \in\right]-s_{0}, s_{0}[$. Then there are constants

$$
\begin{array}{r}
p_{1}=p_{1}\left(s_{0}, \underline{\beta}, \bar{\beta}, a, b\right)=\frac{a}{a+b} \frac{\bar{\beta}}{\underline{\beta}^{2}} e^{2(a+b) s_{0}}, \\
q_{1}=q_{1}\left(s_{0}, \underline{\beta}, \bar{\beta}, a, b, y_{1}-y_{0}\right)=\frac{\bar{\beta}^{2}}{\underline{\beta}^{2}}\left|y_{1}-y_{0}\right|^{2} e^{2(a+b) s_{0}},
\end{array}
$$

such that every geodesic $\gamma=(x, y):[0,1] \rightarrow M$ with $y(0)=y_{0}$ and $y(1)=y_{1}$ and $E_{\alpha, \beta}(x, y)=c$ satisfies

$$
\|y\|_{\infty} \leq s_{0}, \quad\left\|y^{\prime}\right\|_{\infty}^{2} \leq p_{1} c^{+}+q_{1}, \quad c \geq-\frac{1}{2} \bar{\beta} q_{1},
$$

where $c^{+}$denotes the positive part of the real number $c$. 
Proof. Since $X \times]-s, s\left[\right.$ is convex for every $s>s_{0}$ and $\left|y_{0}\right|<s_{0},\left|y_{1}\right|<s_{0}$, we have

$$
\|y\|_{\infty} \leq s_{0}
$$

Let $t_{1} \in[0,1]$ be a point where $\left|y^{\prime}\right|$ attains its maximum. By the mean value theorem, up to inverting the time parameterization, $t \mapsto 1-t$, we may assume that there is $t_{0} \leq t_{1}$ such that $y^{\prime}$ does not change sign on $\left[t_{0}, t_{1}\right]$, and $y^{\prime}\left(t_{0}\right)$ is either 0 or $y_{1}-y_{0}$. Up to considering the change of variable $(x, y) \mapsto(x,-y)$, we may also assume that $y^{\prime} \geq 0$ on $\left[t_{0}, t_{1}\right]$.

By the geodesic equation (6), by (h3), (h4), and by the conservation of energy (7), we have

$$
\begin{aligned}
\left(\beta y^{\prime}\right)^{\prime} & =\frac{1}{2} \partial_{y} \beta\left|y^{\prime}\right|^{2}-\frac{1}{2} g\left[\partial_{y} \alpha x^{\prime}, x^{\prime}\right] \leq \frac{b}{2} \beta\left|y^{\prime}\right|^{2}+\frac{a}{2} g\left[\alpha x^{\prime}, x^{\prime}\right] \\
& =\frac{b}{2} \beta\left|y^{\prime}\right|^{2}+\frac{a}{2} \beta\left|y^{\prime}\right|^{2}+\frac{a}{2} c \leq \frac{a+b}{2} \beta\left|y^{\prime}\right|^{2}+\frac{a}{2} c^{+},
\end{aligned}
$$

where $c^{+}$denotes the positive part of the real number $c$. Then

$$
\left(\beta y^{\prime}\right)^{\prime}-\frac{a+b}{2} \beta\left|y^{\prime}\right|^{2} \leq \frac{a}{2} c^{+},
$$

and multiplying both sides of this inequality by $2 \beta y^{\prime} e^{-(a+b) y}$ we obtain, using also $(\mathrm{h} 2)$,

$$
\left(\beta^{2}\left|y^{\prime}\right|^{2} e^{-(a+b) y}\right)^{\prime} \leq \frac{a}{a+b} c^{+} \beta\left(-e^{-(a+b) y}\right)^{\prime} \leq \frac{a}{a+b} c^{+} \bar{\beta}\left(-e^{-(a+b) y}\right)^{\prime},
$$

on the interval $\left[t_{0}, t_{1}\right]$. Since $y^{\prime}\left(t_{0}\right) \leq\left|y_{1}-y_{0}\right|$, integration on such an interval gives us

$$
\begin{aligned}
& \beta\left(x\left(t_{1}\right), y\left(t_{1}\right)\right)^{2}\left|y^{\prime}\left(t_{1}\right)\right|^{2} e^{-(a+b) y\left(t_{1}\right)} \\
\leq & \beta\left(x\left(t_{0}\right), y\left(t_{0}\right)\right)^{2}\left|y_{1}-y_{0}\right|^{2} e^{-(a+b) y\left(t_{0}\right)}+\frac{a}{a+b} c^{+} \bar{\beta}\left(e^{-(a+b) y\left(t_{0}\right)}-e^{-(a+b) y\left(t_{1}\right)}\right) .
\end{aligned}
$$

By (h2) and (9), we deduce that

$$
\left\|y^{\prime}\right\|_{\infty}^{2}=\left|y^{\prime}\left(t_{1}\right)\right|^{2} \leq\left(\frac{a}{a+b} \frac{\bar{\beta}}{\underline{\beta^{2}}} c^{+}+\frac{\bar{\beta}^{2}}{\underline{\beta}^{2}}\left|y_{1}-y_{0}\right|^{2}\right) e^{2(a+b) s_{0}},
$$

hence

$$
\left\|y^{\prime}\right\|_{\infty}^{2} \leq p_{1} c^{+}+q_{1}
$$

as claimed. In particular, if $c \leq 0$ we have $\left\|y^{\prime}\right\|_{\infty}^{2} \leq q_{1}$. By (h2) this implies

$$
c=E_{\alpha, \beta}(x, y) \geq-\frac{1}{2} \bar{\beta}\left\|y^{\prime}\right\|_{2}^{2} \geq-\frac{1}{2} \bar{\beta}\left\|y^{\prime}\right\|_{\infty}^{2} \geq-\frac{1}{2} \bar{\beta} q_{1},
$$

concluding the proof. $\square$

REMARK 3.2. By Lemma 2.2, if the Lorentzian structure defined by $(\alpha, \beta)$ satisfies (h0), so does the Lorentzian structure defined by $(\alpha, \beta+\lambda)$, for every positive constant 
$\lambda$. Therefore, if $(\alpha, \beta) \in \Gamma\left(s_{0}, \underline{\alpha}, \bar{\alpha}, \beta, \bar{\beta}, a, b\right)$ then $(\alpha, \beta+\lambda) \in \Gamma\left(s_{0}, \underline{\alpha}, \bar{\alpha}, \beta+\lambda, \bar{\beta}+\right.$ $\lambda, a, b)$, for every $\lambda \geq 0$. Notice also that since $\bar{\beta} \geq \underline{\beta}$, the inequalities

$$
\frac{\bar{\beta}+\lambda}{\underline{\beta}+\lambda}=1+\frac{\bar{\beta}-\underline{\beta}}{\underline{\beta}+\lambda} \leq 1+\frac{\bar{\beta}-\underline{\beta}}{\underline{\beta}}=\frac{\bar{\beta}}{\underline{\beta}}, \quad \frac{\bar{\beta}+\lambda}{(\underline{\beta}+\lambda)^{2}} \leq \frac{\bar{\beta}}{\underline{\beta}} \frac{1}{\underline{\beta}+\lambda} \leq \frac{\bar{\beta}}{\underline{\beta}^{2}},
$$

hold for any $\lambda \geq 0$. We conclude that

$$
\begin{array}{r}
p_{1}\left(s_{0}, \underline{\beta}+\lambda, \bar{\beta}+\lambda, a, b\right) \leq p_{1}\left(s_{0}, \underline{\beta}, \bar{\beta}, a, b\right), \\
q_{1}\left(s_{0}, \underline{\beta}+\lambda, \bar{\beta}+\lambda, a, b, y_{1}-y_{0}\right) \leq q_{1}\left(s_{0}, \underline{\beta}, \bar{\beta}, a, b, y_{1}-y_{0}\right),
\end{array}
$$

for every positive constant $\lambda$.

Lemma 3.1 has the following consequence:

Lemma 3.3. Let $(\alpha, \beta) \in \Gamma\left(s_{0}, \underline{\alpha}, \bar{\alpha}, \underline{\beta}, \bar{\beta}, a, b\right)$, and let $\left.y_{0}, y_{1} \in\right]-s_{0}, s_{0}[$. For every $\lambda$ in the interval $\left[0,2 / p_{1}\left(s_{0}, \underline{\beta}, \bar{\beta}, a, b\right)\right]$, for every $\mu \in[0, \lambda]$, and for every critical point $(x, y)$ of $E_{\alpha, \beta+\mu}$, there holds

$$
E_{\alpha, \beta+\lambda}(x, y) \geq-\frac{1}{2}(\bar{\beta}+\lambda) q_{1}\left(s_{0}, \underline{\beta}, \bar{\beta}, a, b, y_{1}-y_{0}\right) .
$$

Proof. Let $(x, y)$ be a critical point of $E_{\alpha, \beta+\mu}$, and notice that

$$
E_{\alpha, \beta+\lambda}(x, y)=E_{\alpha, \beta+\mu}(x, y)-\frac{1}{2}(\lambda-\mu)\left\|y^{\prime}\right\|_{2}^{2} .
$$

If $E_{\alpha, \beta+\mu}(x, y) \leq 0$, by Lemma 3.1 we have

$E_{\alpha, \beta+\mu}(x, y)-\frac{1}{2}(\lambda-\mu)\left\|y^{\prime}\right\|_{2}^{2} \geq-\frac{1}{2}(\bar{\beta}+\mu) q_{1}(\mu)-\frac{1}{2}(\lambda-\mu) q_{1}(\mu)=-\frac{1}{2}(\bar{\beta}+\lambda) q_{1}(\mu)$,

where $q_{1}(\mu)$ stays for $q_{1}\left(s_{0}, \underline{\beta}+\mu, \bar{\beta}+\mu, a, b, y_{1}-y_{0}\right)$. By Remark 3.2, $q_{1}(\mu) \leq q_{1}(0)$, so (10) and (11) imply

$$
E_{\alpha, \beta+\lambda}(x, y) \geq-\frac{1}{2}(\bar{\beta}+\lambda) q_{1}(0) .
$$

If $E_{\alpha, \beta+\mu}(x, y)>0$, by Lemma 3.1 we have

$$
\begin{array}{r}
E_{\alpha, \beta+\mu}(x, y)-\frac{1}{2}(\lambda-\mu)\left\|y^{\prime}\right\|_{2}^{2} \geq E_{\alpha, \beta+\mu}(x, y)-\frac{1}{2}(\lambda-\mu) p_{1}(\mu) E_{\alpha, \beta+\mu}(x, y) \\
-\frac{1}{2}(\lambda-\mu) q_{1}(\mu) \geq\left(1-\frac{1}{2}(\lambda-\mu) p_{1}(\mu)\right) E_{\alpha, \beta+\mu}(x, y)-\frac{1}{2} \lambda q_{1}(0),
\end{array}
$$

where $p_{1}(\mu)$ stays for $p_{1}\left(s_{0}, \underline{\beta}+\mu, \bar{\beta}+\mu, a, b\right)$ and we have used again the inequality $q_{1}(\mu) \leq q_{1}(0)$. By Remark $3 . \overline{2}$ we also have $p_{1}(\mu) \leq p_{1}(0)$, so

$$
\frac{1}{2}(\lambda-\mu) p_{1}(\mu) \leq \frac{1}{2} \lambda p_{1}(0) \leq 1
$$

by the assumption on $\lambda$. Therefore, (10) and (13) imply

$$
E_{\alpha, \beta+\lambda}(x, y) \geq-\frac{1}{2} \lambda q_{1}(0) \geq-\frac{1}{2}(\bar{\beta}+\lambda) q_{1}(0) .
$$

We conclude that every critical point $(x, y)$ of $E_{\alpha, \beta+\mu}$ satisfies (12), as claimed. 
4. The $\mathbf{W}^{1,2}$ setting for the energy functional. Let $\mathscr{X}$ be the Hilbert manifold of paths $x:[0,1] \rightarrow X$ of Sobolev class $W^{1,2}$, such that $x(0)=x_{0}$ and $x(1)=x_{1}$. Let $\mathscr{Y}$ be the affine Hilbert manifold

$$
\mathscr{Y}=\left\{y \in W^{1,2}([0,1], \mathbb{R}) \mid y(0)=y_{0}, y(1)=y_{1}\right\}
$$

modeled on the Hilbert space $H=W_{0}^{1,2}([0,1], \mathbb{R})$. The space $\mathscr{M}$ of $W^{1,2}$ paths in $M$ joining $\left(x_{0}, y_{0}\right)$ to $\left(x_{1}, y_{1}\right)$ is then the product manifold $\mathscr{M}=\mathscr{X} \times \mathscr{Y}$. The energy functional $E_{\alpha, \beta}$ is smooth on $\mathscr{M}$.

On $\mathscr{M}=\mathscr{X} \times \mathscr{Y}$ we consider the Riemannian structure given by

$$
\left\langle\left(\xi_{1}, \eta_{1}\right),\left(\xi_{2}, \eta_{2}\right)\right\rangle:=\int_{0}^{1} g\left(\nabla_{t}^{g} \xi_{1}, \nabla_{t}^{g} \xi_{2}\right) d t+\int_{0}^{1} \eta_{1}^{\prime} \eta_{2}^{\prime} d t
$$

where $\xi_{1}, \xi_{2} \in T_{x} \mathscr{X}$ are $W_{0}^{1,2}$ vector fields along $x \in \mathscr{X}, \nabla_{t}^{g}$ denotes the Levi-Civita covariant derivative along $x$ induced by the metric $g$, and $\eta_{1}, \eta_{2} \in H$. The associated norm is denoted by $\|\cdot\|$, and the $\langle\cdot, \cdot\rangle$-gradient of a functional $f: \mathscr{M} \rightarrow \mathbb{R}$ is denoted by $\nabla f$. The Riemannian metric (14) induces a complete distance on $\mathscr{M}$, and a subset $\mathscr{A}$ of $\mathscr{M}$ is bounded if and only if there is some number $c$ such that

$$
\int_{0}^{1} g\left(x^{\prime}, x^{\prime}\right) d t \leq c, \quad\left\|y^{\prime}\right\|_{2} \leq c
$$

for every $(x, y) \in \mathscr{A},\|\cdot\|_{2}$ denoting the $L^{2}$ norm.

LEMMA 4.1. Let $z=(x, y)$ be a critical point of $E_{\alpha, \beta}$. Then the positive eigenspace (resp. the negative eigenspace) of the Hessian of $E_{\alpha, \beta}$ at $z$ is a compact perturbation of $T_{x} \mathscr{X} \times(0)$ (resp. of $\left.(0) \times T_{y} \mathscr{Y}=(0) \times H\right)$.

Proof. The second differential of the energy functional $E_{\alpha, \beta}$ at a critical point $z=(x, y)$ is given by the expression

$$
D^{2} E_{\alpha, \beta}(x, y)\left[\zeta_{1}, \zeta_{2}\right]=\int_{0}^{1}\left[h\left(\nabla_{t}^{h} \zeta_{1}, \nabla_{t}^{h} \zeta_{2}\right)-h\left(R\left(\zeta_{1}, z^{\prime}\right) z^{\prime}, \zeta_{2}\right)\right] d t
$$

for every $\zeta_{1}, \zeta_{2} \in T_{z} \mathscr{M}$, where $\nabla_{t}^{h}$ denotes the Levi-Civita covariant derivative along the curve $z$ associated to the Lorentzian structure $h$, and $R$ is the Riemann tensor of $h$ (see for instance [O'N83], Chapter 10, Proposition 39). Since any two covariant derivatives along a fixed curve $z$ differ by a tensor, for every $\zeta=(\xi, \eta) \in T_{x} \mathscr{X} \times H=$ $T_{z} \mathscr{M}$ we have

$$
\nabla_{t}^{h} \zeta(t)=\left(\nabla_{t}^{g} \xi(t)+\Gamma_{X}(t)[\zeta(t)], \eta^{\prime}(t)+\Gamma_{Y}(t)[\zeta(t)]\right), \quad \forall t \in[0,1]
$$

for some $(1,1)$-tensor $\Gamma_{X} \oplus \Gamma_{Y}$ on $z^{*}(T M)$. Therefore, writing $\zeta_{1}=\left(\xi_{1}, \eta_{1}\right), \zeta_{2}=$ $\left(\xi_{2}, \eta_{2}\right)$, with $\xi_{1}, \xi_{2} \in T_{x} \mathscr{X}, \eta_{1}, \eta_{2} \in H$, we have

$$
\begin{aligned}
& D^{2} E_{\alpha, \beta}(z)\left[\zeta_{1}, \zeta_{2}\right]=\int_{0}^{1}\left[g\left(\alpha(z) \nabla_{t}^{g} \xi_{1}, \nabla_{t}^{g} \xi_{2}\right)-\beta(z) \eta_{1}^{\prime} \eta_{2}^{\prime}\right] d t \\
& +\int_{0}^{1}\left[g\left(\alpha(z) \nabla_{t}^{g} \xi_{1}, \Gamma_{X}\left[\zeta_{2}\right]\right)+g\left(\alpha(z) \Gamma_{X}\left[\zeta_{1}\right], \nabla_{t}^{g} \xi_{2}\right)+g\left(\alpha(z) \Gamma_{X}\left[\zeta_{1}\right], \Gamma_{X}\left[\zeta_{2}\right]\right)\right. \\
& \left.-\beta(z) \eta_{1}^{\prime} \Gamma_{Y}\left[\zeta_{2}\right]-\beta(z) \Gamma_{Y}\left[\zeta_{1}\right] \eta_{2}^{\prime}-\beta(z) \Gamma_{Y}\left[\zeta_{1}\right] \Gamma_{Y}\left[\zeta_{2}\right]-h\left(R\left(\zeta_{1}, z^{\prime}\right) z^{\prime}, \zeta_{2}\right)\right] d t
\end{aligned}
$$


The first integral in the above formula defines a symmetric continuous bilinear form on $T_{z} \mathscr{M}$, represented by an invertible self-adjoint operator $T \in L\left(T_{z} \mathscr{M}, T_{z} \mathscr{M}\right)$, whose positive and negative eigenspaces are $T_{x} \mathscr{X} \times(0)$ and $(0) \times H$, respectively. By the compactness of the embedding $W^{1,2} \hookrightarrow L^{2}$, the second integral in the above formula defines a symmetric bilinear form which is continuous with respect to the weak topology of $T_{z} \mathscr{M}$, hence it is represented by a compact self-adjoint operator $K \in L\left(T_{z} \mathscr{M}, T_{z} \mathscr{M}\right)$.

Then $\nabla^{2} E_{\alpha, \beta}(z)=T+K$, and the conclusion follows from the fact that the positive (resp. negative) eigenspace of a compact perturbation of a self-adjoint invertible operator $T$ is a compact perturbation of the positive (resp. negative) eigenspace of $T$ (see [AM01], Proposition 2.2).

Therefore, the relative Morse index

$$
i_{\text {rel }}(z)=\operatorname{ind}\left(V^{-}\left(\nabla^{2} E_{\alpha, \beta}(z)\right), T_{x} \mathscr{X} \times(0)\right)
$$

introduced in (5) is a well-defined integer.

Lemma 4.2. Let $\left(\alpha_{0}, \beta_{0}\right)$ and $\left(\alpha_{1}, \beta_{1}\right)$ be Lorentzian structures in $\Gamma\left(s_{0}, \underline{\alpha}, \bar{\alpha}, \underline{\beta}, \bar{\beta}, a, b\right)$ such that

$$
\max \left\{\left\|\alpha_{0}-\beta_{0}\right\|_{\infty},\left\|\beta_{1}-\beta_{0}\right\|_{\infty}\right\}<\frac{\lambda \underline{\alpha}}{\underline{\alpha}+\bar{\beta}},
$$

for some $\lambda>0$. Then for every $c_{0}, c_{1} \in \mathbb{R}$ the set

$$
\left\{E_{\alpha_{0}, \beta_{0}} \leq c_{0}\right\} \cap\left\{E_{\alpha_{1}, \beta_{1}+\lambda} \geq c_{1}\right\}
$$

is bounded in $\mathscr{M}$.

Proof. Let $(x, y) \in\left\{E_{\alpha_{0}, \beta_{0}} \leq c_{0}\right\} \cap\left\{E_{\alpha_{1}, \beta_{1}+\lambda} \geq c_{1}\right\}$. Then

$$
\begin{aligned}
& \int_{0}^{1} g\left(x^{\prime}, x^{\prime}\right) d t \leq \frac{1}{\underline{\alpha}} \int_{0}^{1} g\left(\alpha_{0} x^{\prime}, x^{\prime}\right) d t \\
= & \frac{2}{\underline{\alpha}} E_{\alpha_{0}, \beta_{0}}(x, y)+\frac{1}{\underline{\alpha}} \int_{0}^{1} \beta_{0}\left|y^{\prime}\right|^{2} d t \leq \frac{2}{\underline{\alpha}} c_{0}+\frac{\bar{\beta}}{\underline{\alpha}}\left\|y^{\prime}\right\|_{2}^{2} .
\end{aligned}
$$

Therefore,

$$
\begin{array}{r}
2 c_{1} \leq 2 E_{\alpha_{1}, \beta_{1}+\lambda}(x, y)=\int_{0}^{1} g\left(\alpha_{1} x^{\prime}, x^{\prime}\right) d t-\int_{0}^{1} \beta_{1}\left|y^{\prime}\right|^{2} d t-\lambda\left\|y^{\prime}\right\|_{2}^{2} \\
\leq 2 E_{\alpha_{0}, \beta_{0}}(x, y)+\left\|\alpha_{1}-\alpha_{0}\right\|_{\infty} \int_{0}^{1} g\left(x^{\prime}, x^{\prime}\right) d t-\left(\lambda-\left\|\beta_{1}-\beta_{0}\right\|_{\infty}\right)\left\|y^{\prime}\right\|_{2}^{2} \\
\leq 2 c_{0}\left(1+\frac{\left\|\alpha_{1}-\alpha_{0}\right\|_{\infty}}{\underline{\alpha}}\right)-\left(\lambda-\left\|\beta_{1}-\beta_{0}\right\|_{\infty}-\frac{\bar{\beta}}{\underline{\alpha}}\left\|\alpha_{1}-\alpha_{0}\right\|_{\infty}\right)\left\|y^{\prime}\right\|_{2}^{2} .
\end{array}
$$

By our assumption on $\left\|\alpha_{1}-\alpha_{0}\right\|_{\infty}$ and $\left\|\beta_{1}-\beta_{0}\right\|_{\infty}$, the coefficient of $\left\|y^{\prime}\right\|_{2}^{2}$ in the latter expression is positive, so the above inequality implies that $\left\|y^{\prime}\right\|_{2}$ is uniformly bounded. By (15), also $\int_{0}^{1} g\left(x^{\prime}, x^{\prime}\right) d t$ is uniformly bounded, hence $(x, y)$ is uniformly bounded in $\mathscr{M}$. $\mathrm{Q}$

The following result is well known (see for instance [BF94], Lemma 3.2), but we include a proof for sake of completeness: 
Lemma 4.3. Assume that $\alpha$ and $\beta$ are strictly positive (the first object as a section over $M$ of $g$-symmetric $(1,1)$ tensors on $X$, the second as a real function on $M)$. Then every bounded sequence $\left(z_{h}\right) \subset \mathscr{M}$ such that $\left\|\nabla E_{\alpha, \beta}\left(z_{h}\right)\right\| \rightarrow 0$ has a converging subsequence.

Proof. Let $\left(z_{h}\right)=\left(x_{h}, y_{h}\right)$ be a bounded sequence in $\mathscr{M}$. By the compactness of the embedding of $W^{1,2}$ into the space of continuous curves, up to a subsequence we may assume that $\left(z_{h}\right)$ converges uniformly to some $\bar{z}=(\bar{x}, \bar{y}) \in \mathscr{M}$. Let $U$ be an open neighborhood of 0 in $\mathbb{R}^{n}$, with $n=\operatorname{dim} X$, and let

$$
[0,1] \times U \rightarrow[0,1] \times X, \quad(t, \xi) \mapsto(t, \varphi(t, \xi)),
$$

be a smooth coordinate system such that $\varphi(0,0)=x_{0}, \varphi(1,0)=x_{1}$, and $\bar{x}(t)=$ $\varphi(t, \bar{\xi}(t))$ for every $t \in[0,1]$, for some $\bar{\xi} \in W_{0}^{1,2}([0,1], U)$. For instance, such a diffeomorphism can be constructed by choosing a smooth curve $x:[0,1] \rightarrow X$ connecting $x_{0}$ and $x_{1}$, with dist $(x(t), \bar{x}(t))<\rho$ for every $t \in[0,1], \rho$ being the injectivity radius of a large compact neighborhood of $\bar{x}([0,1])$ in $X$, and by setting

$$
\varphi(t, \xi):=\exp _{x(t)}(\Phi(t) \xi)
$$

where $\xi \in \mathbb{R}^{n},|\xi|<\rho$, and $\Phi$ is a smooth orthogonal trivialization of the vector bundle $x^{*}(T X)$ over $[0,1]$.

Then the map

$$
\varphi_{*}: W_{0}^{1,2}([0,1], U) \rightarrow \mathscr{X}, \quad \varphi_{*}(\xi)(t):=\varphi(t, \xi(t)),
$$

is a smooth local coordinate system on the Hilbert manifold $\mathscr{X}$. Up to replacing $U$ by a smaller neighborhood, we may assume that $D \varphi_{*}$ is bounded together with its inverse from the standard metric of $W_{0}^{1,2}\left([0,1], \mathbb{R}^{n}\right)$ to the Riemannian metric of $\mathscr{X}$ defined in (14).

Since $\left(x_{h}\right)$ converges uniformly to $\bar{x}$, it eventually belongs to the image of $\varphi_{*}$, so up to a subsequence we may assume that $\varphi_{*}\left(\xi_{h}\right)=x_{h}$ for some $\left(\xi_{h}\right) \subset W_{0}^{1,2}([0,1], U)$ converging to $\bar{\xi}$ uniformly and weakly in $W_{0}^{1,2}\left([0,1], \mathbb{R}^{n}\right)$. By the properties of $\varphi_{*}$, the fact that $\left\|\nabla E_{\alpha, \beta}\left(z_{n}\right)\right\|$ is infinitesimal is equivalent to the fact that, setting

$$
\tilde{E}(\xi, y):=E_{\alpha, \beta}\left(\varphi_{*}(\xi), y\right), \quad \forall \xi \in W_{0}^{1,2}([0,1], U), \forall y \in \mathscr{Y},
$$

the sequence $\left(D \tilde{E}\left(\xi_{h}, y_{h}\right)\right)$ is infinitesimal in the dual of $W_{0}^{1,2}\left([0,1], \mathbb{R}^{n}\right) \times$ $W_{0}^{1,2}([0,1], \mathbb{R})$. It is sufficient to show that $\left(\xi_{h}, y_{h}\right)$ converges to $(\bar{\xi}, \bar{y})$ strongly in $W^{1,2}$, because this fact is equivalent to the convergence of $\left(x_{h}, y_{h}\right)$ to $(\bar{x}, \bar{y})$ in $\mathscr{M}$.

A direct computation shows that $\tilde{E}$ has the form

$\tilde{E}(\xi, y)=\frac{1}{2} \int_{0}^{1}\left[A(t, \xi, y) \xi^{\prime} \cdot \xi^{\prime}+B(t, \xi, y) \cdot \xi^{\prime}+C(t, \xi, y)\right] d t-\frac{1}{2} \int_{0}^{1} \tilde{\beta}(t, \xi, y)\left|y^{\prime}\right|^{2} d t$,

where $A$ takes value in the space of positive definite symmetric $n \times n$ matrices, and $\tilde{\beta}$ is strictly positive. Since $\left(\xi_{h}-\bar{\xi}\right)$ is bounded in $W_{0}^{1,2}$, the sequence $D \tilde{E}\left(\xi_{h}, y_{h}\right)\left[\left(\xi_{h}-\bar{\xi}, 0\right)\right]$ is infinitesimal. From the expression

$$
\begin{aligned}
& D \tilde{E}\left(\xi_{h}, y_{h}\right)\left[\left(\xi_{h}-\bar{\xi}, 0\right)\right] \\
= & \int_{0}^{1} A\left(t, \xi_{h}, y_{h}\right) \xi_{h}^{\prime} \cdot\left(\xi_{h}^{\prime}-\bar{\xi}^{\prime}\right) d t+\frac{1}{2} \int_{0}^{1}\left[D_{2} A\left(t, \xi_{h}, y_{h}\right)\left[\xi_{h}-\bar{\xi}\right] \xi_{h}^{\prime} \cdot \xi_{h}^{\prime}\right. \\
& +B\left(t, \xi_{h}, y_{h}\right) \cdot\left(\xi_{h}^{\prime}-\bar{\xi}^{\prime}\right)+D_{2} B\left(t, \xi_{h}, y_{h}\right)\left[\xi_{h}-\bar{\xi}\right] \cdot \xi_{h}^{\prime}+D_{2} C\left(t, \xi_{h}, y_{h}\right)\left[\xi_{h}-\bar{\xi}\right] \\
& \left.-D_{2} \tilde{\beta}\left(t, \xi_{h}, y_{h}\right)\left[\xi_{h}-\bar{\xi}\right]\left|y_{h}^{\prime}\right|^{2}\right] d t,
\end{aligned}
$$


and from the fact that $\xi_{h} \rightarrow \bar{\xi}$ uniformly and $\xi_{h}^{\prime} \rightarrow \bar{\xi}^{\prime}$ weakly in $L^{2}$, we deduce that the first integral above is infinitesimal. Therefore, also

$$
\int_{0}^{1} A\left(t, \xi_{h}, y_{h}\right)\left(\xi_{h}^{\prime}-\bar{\xi}^{\prime}\right) \cdot\left(\xi_{h}^{\prime}-\bar{\xi}^{\prime}\right) d t
$$

is infinitesimal, and the fact that $A$ is positive definite implies that $\xi_{h} \rightarrow \bar{\xi}$ in $W^{1,2}$. Similarly, $D \tilde{E}\left(\xi_{h}, y_{h}\right)\left[\left(0, y_{h}-\bar{y}\right)\right]$ is infinitesimal, so the last integral in the expression

$$
\begin{aligned}
& D \tilde{E}\left(\xi_{h}, y_{h}\right)\left[\left(0, y_{h}-\bar{y}\right)\right] \\
= & \frac{1}{2} \int_{0}^{1}\left[D_{3} A\left(t, \xi_{h}, y_{h}\right)\left[y_{h}-\bar{y}\right] \xi_{h}^{\prime} \cdot \xi_{h}^{\prime}+D_{3} B\left(t, \xi_{h}, y_{h}\right)\left[y_{h}-\bar{y}\right] \cdot \xi_{h}^{\prime}\right. \\
& \left.+D_{3} C\left(t, \xi_{h}, y_{h}\right)\left[y_{h}-\bar{y}\right]-D_{3} \tilde{\beta}\left(t, \xi_{h}, y_{h}\right)\left[y_{h}-\bar{y}\right]\left|y_{h}^{\prime}\right|^{2}\right] d t \\
& -\int_{0}^{1} \tilde{\beta}\left(t, \xi_{h}, y_{h}\right) y_{h}^{\prime}\left(y_{h}^{\prime}-\bar{y}^{\prime}\right) d t
\end{aligned}
$$

is infinitesimal. Therefore, also

$$
\int_{0}^{1} \tilde{\beta}\left(t, \xi_{h}, y_{h}\right)\left|y_{h}^{\prime}-\bar{y}^{\prime}\right|^{2} d t
$$

is infinitesimal, and the fact that $\tilde{\beta}$ is strictly positive implies that $\left(y_{h}-\bar{y}\right)$ converges to 0 in $W^{1,2}$, concluding the proof.

5. A pseudo-gradient vector field for the energy functional. Let $\chi$ be a smooth real function on $\mathbb{R}$ such that $\chi(s)=0$ for $s \leq 0, \chi(s)=1$ for $s \geq 1$, and $\chi^{\prime} \geq 0$. We choose two numbers

$$
\lambda:=\frac{2}{p_{1}\left(s_{0}, \underline{\beta}, \bar{\beta}, a, b\right)}, \quad c_{0}<-\frac{1}{2}(\bar{\beta}+\lambda) q_{1}\left(s_{0}, \underline{\beta}, \bar{\beta}, a, b, y_{1}-y_{0}\right) .
$$

By Lemma 3.3 with $\mu=0$,

$$
\operatorname{crit} E_{\alpha, \beta} \subset\left\{E_{\alpha, \beta+\lambda}>c_{0}\right\} .
$$

By the same lemma with $\mu=\lambda$, the manifold $\mathscr{M}$ is covered by the open sets $\left\{\nabla E_{\alpha, \beta+\lambda} \neq 0\right\}$ and $\left\{E_{\alpha, \beta+\lambda}>c_{0}\right\}$. Therefore, the formula

$$
-G=\nabla E_{\alpha, \beta}+\chi\left(c_{0}-E_{\alpha, \beta+\lambda}\right) \frac{\left\|\nabla E_{\alpha, \beta}\right\|}{\left\|\nabla E_{\alpha, \beta+\lambda}\right\|} \nabla E_{\alpha, \beta+\lambda}
$$

defines a smooth vector field $G$ on $\mathscr{M}$, which coincides with $-\nabla E_{\alpha, \beta}$ on $\left\{E_{\alpha, \beta+\lambda}>\right.$ $\left.c_{0}\right\}$. It is useful to make $G$ bounded, by multiplying it for a suitable smooth positive function, obtaining the smooth bounded vector field

$$
F:=\frac{G}{\sqrt{1+\|G\|^{2}}}
$$

LEMMA 5.1.

(i) $F$ is a Morse vector field, and $\operatorname{rest} F=\operatorname{crit} E_{\alpha, \beta}$;

(ii) $E_{\alpha, \beta}$ is a non-degenerate Lyapunov function for $F$ on $\mathscr{M}$; 
(iii) $E_{\alpha, \beta+\mu}$ is a Lyapunov function for $F$ on $\left\{E_{\alpha, \beta+\lambda}<c_{0}-1\right\}$ (where $F$ has no rest points).

Proof. Since the function $\left(1+\|G\|^{2}\right)^{-1 / 2}$ is positive, it is enough to prove (i), (ii), and (iii) for the vector field $G$. The numbers $D E_{\alpha, \beta}[-G]$ and $D E_{\alpha, \beta+\lambda}[-G]$ are

$$
\begin{aligned}
D E_{\alpha, \beta}[-G] & =\left\langle\nabla E_{\alpha, \beta},-G\right\rangle \\
& =\left\|\nabla E_{\alpha, \beta}\right\|^{2}+\chi\left(c_{0}-E_{\alpha, \beta+\lambda}\right) \frac{\left\|\nabla E_{\alpha, \beta}\right\|}{\left\|\nabla E_{\alpha, \beta+\lambda}\right\|}\left\langle\nabla E_{\alpha, \beta}, \nabla E_{\alpha, \beta+\lambda}\right\rangle, \\
D E_{\alpha, \beta+\lambda}[-G] & =\left\langle\nabla E_{\alpha, \beta+\lambda},-G\right\rangle \\
& =\left\langle\nabla E_{\alpha, \beta}, \nabla E_{\alpha, \beta+\lambda}\right\rangle+\chi\left(c_{0}-E_{\alpha, \beta+\lambda}\right)\left\|\nabla E_{\alpha, \beta}\right\|\left\|\nabla E_{\alpha, \beta+\lambda}\right\| .
\end{aligned}
$$

Since $0 \leq \chi \leq 1$, by the Cauchy-Schwarz inequality the quantity (17) is greater or equal than zero, and it equals zero if and only if $\nabla E_{\alpha, \beta}=0$ or

$$
\chi\left(c_{0}-E_{\alpha, \beta+\lambda}\right)=1 \quad \text { and } \quad\left\langle\nabla E_{\alpha, \beta}, \nabla E_{\alpha, \beta+\lambda}\right\rangle=-\left\|\nabla E_{\alpha, \beta}\right\|\left\|\nabla E_{\alpha, \beta+\lambda}\right\| .
$$

The latter cannot occur, for it implies $\nabla E_{\alpha, \beta+\lambda}=-\nu \nabla E_{\alpha, \beta}$ with $\nu \geq 0$, that is $\nabla E_{\alpha, \beta+\lambda /(1+\nu)}=0$, so by Lemma 3.3 and by our choice of $c_{0}, E_{\alpha, \beta+\lambda}>c_{0}$, hence $\chi\left(c_{0}-E_{\alpha, \beta+\lambda}\right)=0$.

This shows that $D E_{\alpha, \beta}[G] \leq 0$, and it equals zero exactly on the critical set of $E_{\alpha, \beta}$. Claims (i) and (ii) follow from the fact that $G=-\nabla E_{\alpha, \beta}$ on a neighborhood of such a set, and $E_{\alpha, \beta}$ is a Morse function.

On the set $\left\{E_{\alpha, \beta+\lambda}<c_{0}-1\right\}$ the value of $\chi\left(c_{0}-E_{\alpha, \beta+\lambda}\right)$ is 1 , so by the CauchySchwarz inequality the quantity (18) is greater or equal than zero, and it cannot vanish because as observed, (19) cannot occur. Therefore $E_{\alpha, \beta+\lambda}$ is a Lyapunov function for $G$ on $\left\{E_{\alpha, \beta+\lambda}<c_{0}-1\right\}$. This proves (iii).

Denote by $P: T \mathscr{M} \rightarrow H$ the map

$$
(x, y, \xi, \eta) \mapsto \eta, \quad \forall(x, y) \in \mathscr{X} \times \mathscr{Y}, \forall \xi \in T_{x} \mathscr{X}, \forall \eta \in T_{y} \mathscr{Y}=H,
$$

that is the differential of the projection of $\mathscr{X} \times \mathscr{Y}$ onto the second factor.

LEMMA 5.2.

(i) The vector field $F$ is Lipschitz continuous on bounded subsets of $\mathscr{M}$.

(ii) For every $y \in \mathscr{Y}$, the map $\mathscr{X} \rightarrow H, x \mapsto P F(x, y)$, is compact.

Proof. Let $\mathscr{A}$ be a bounded subset of $\mathscr{M}$. The vector fields $\nabla E_{\alpha, \beta}$ and $\nabla E_{\alpha, \beta+\lambda}$ are readily seen to be Lipschitz continuous on $\mathscr{A}$. The function $\chi\left(c_{0}-\right.$ $\left.E_{\alpha, \beta+\lambda}\right) /\left\|\nabla E_{\alpha, \beta+\lambda}\right\|$ is also Lipschitz on $\mathscr{A}$. Indeed, it is enough to check that $\left\|\nabla E_{\alpha, \beta+\lambda}\right\|$ is bounded away from zero on $\mathscr{A} \cap\left\{\chi\left(c_{0}-E_{\alpha, \beta+\lambda}\right)>0\right\}$, and this follows from Lemma 4.3 and from the fact that $E_{\alpha, \beta+\lambda}$ has no critical points in $\left\{E_{\alpha, \beta+\lambda} \leq c_{0}\right\}$ (again by Lemma 3.3 with $\mu=\lambda$ ). We conclude that $G$ is Lipschitz continuous on $B$, and so is $F$. This proves (i).

Let $\mathscr{X}_{0}$ be a bounded subset of $\mathscr{X}$, and let $y \in \mathscr{Y}$. We must show that $\operatorname{PF}\left(\mathscr{X}_{0} \times\{y\}\right)$ has compact closure in $H$. Since $\chi\left(c_{0}-E_{\alpha, \beta+\lambda}\right)\left\|\nabla E_{\alpha, \beta}\right\| /\left\|\nabla E_{\alpha, \beta+\lambda}\right\|$ is bounded on bounded sets, it is enough to show that $P \nabla E_{\alpha, \beta}\left(\mathscr{X}_{0} \times\{y\}\right.$ ) (and similarly $\left.P \nabla E_{\alpha, \beta+\lambda}\left(\mathscr{X}_{0} \times\{y\}\right)\right)$ has compact closure in $H$. Equivalently, we must show that if $\left(x_{n}\right) \subset \mathscr{X}_{0}$, and $\left(v_{n}\right) \subset H$ converges weakly to 0 , then

$$
\left\langle P \nabla E_{\alpha, \beta}\left(x_{n}, y\right), v_{n}\right\rangle \rightarrow 0 .
$$


Up to a subsequence, we may assume that $x_{n}$ converges uniformly to some $x \in \mathscr{X}$, and that $v_{n}$ converges uniformly to 0 . Now,

$$
\begin{array}{r}
\left\langle P \nabla E_{\alpha, \beta}\left(x_{n}, y\right), v_{n}\right\rangle=\left\langle\nabla E_{\alpha, \beta}\left(x_{n}, y\right), v_{n}\right\rangle=D E_{\alpha, \beta}\left(x_{n}, y\right)\left[v_{n}\right] \\
=\frac{1}{2} \int_{0}^{1} g\left(\partial_{y} \alpha\left(x_{n}, y\right) x_{n}^{\prime}, x_{n}^{\prime}\right) v_{n} d t-\frac{1}{2} \int_{0}^{1} \partial_{y} \beta\left(x_{n}, y\right)\left|y^{\prime}\right|^{2} v_{n} d t-\int_{0}^{1} \beta\left(x_{n}, y\right) y^{\prime} v_{n}^{\prime} d t .
\end{array}
$$

The sequences $g\left(\partial_{y} \alpha\left(x_{n}, y\right) x_{n}^{\prime}, x_{n}^{\prime}\right)$ and $\partial_{y} \beta\left(x_{n}, y\right)\left|y^{\prime}\right|^{2}$ are bounded in $L^{1}$, so the first two integrals are infinitesimal. The sequence $\beta\left(x_{n}, y\right)$ is bounded in $L^{\infty}$, so the third integral is also infinitesimal. This proves (ii).

The proof of the Palais-Smale property for $\left(F, E_{\alpha, \beta}\right)$ on bounded subsets of $\mathscr{M}$ makes use of the above lemma, together with the following elementary inequality for vectors in a Hilbert space. Then

Lemma 5.3. Let $u$ and $v \neq 0$ be vectors in a Hilbert space, and let $\chi \in[0,1]$.

$$
\|u\|^{2}+\chi \frac{\|u\|}{\|v\|}\langle u, v\rangle \geq \frac{1}{2} \min _{0 \leq \theta \leq 1}\|\theta v+(1-\theta) u\|^{2} .
$$

Proof. If $\langle u, v\rangle \geq 0$ the inequality holds trivially, by choosing $\theta=0$. So we assume $\langle u, v\rangle<0$. In this case, $\|u-v\| \neq 0$ and

$$
0 \leq\langle u, u-v\rangle=\|u\|^{2}-\langle u, v\rangle \leq\|u\|^{2}-2\langle u, v\rangle+\|v\|^{2}=\|u-v\|^{2},
$$

so the number $\langle u, u-v\rangle /\|u-v\|^{2}$ is in $[0,1]$. Therefore,

$$
\begin{aligned}
\min _{0 \leq \theta \leq 1}\|\theta v+(1-\theta) u\|^{2}=\min _{0 \leq \theta \leq 1}\|u-\theta(u-v)\|^{2} & \leq\left\|u-\frac{\langle u, u-v\rangle}{\|u-v\|^{2}}(u-v)\right\|^{2} \\
& =\|u\|^{2}-\frac{\langle u, u-v\rangle^{2}}{\|u-v\|^{2}} .
\end{aligned}
$$

Moreover,

$$
\begin{array}{r}
\|u\|^{2}-\frac{\langle u, u-v\rangle^{2}}{\|u-v\|^{2}}=\|u\|^{2}\left(1+\left\langle\frac{u}{\|u\|}, \frac{u-v}{\|u-v\|}\right\rangle\right)\left(1-\left\langle\frac{u}{\|u\|}, \frac{u-v}{\|u-v\|}\right\rangle\right) \\
\leq 2\|u\|^{2}\left(1-\left\langle\frac{u}{\|u\|}, \frac{u-v}{\|u-v\|}\right\rangle\right) \leq 2\|u\|^{2}\left(1+\left\langle\frac{u}{\|u\|}, \frac{v}{\|v\|}\right\rangle\right),
\end{array}
$$

the latter inequality following from

$$
-\langle u, v\rangle\|u-v\| \leq\left|\|u\|^{2}-\langle u, v\rangle\right|\|v\|,
$$

which is easily verified by taking the squares and using the Cauchy-Schwarz inequality. By (20) and (21),

$$
\min _{0 \leq \theta \leq 1}\|\theta v+(1-\theta) u\|^{2} \leq 2\left(\|u\|^{2}+\frac{\|u\|}{\|v\|}\langle u, v\rangle\right) \leq 2\left(\|u\|^{2}+\chi \frac{\|u\|}{\|v\|}\langle u, v\rangle\right),
$$

because $\langle u, v\rangle<0$ and $\chi \leq 1$. This concludes the proof. $\square$

Lemma 5.4. Every bounded sequence $z_{n}=\left(x_{n}, y_{n}\right) \in \mathscr{M}$ such that $D E_{\alpha, \beta}\left(z_{n}\right)\left[F\left(z_{n}\right)\right] \rightarrow 0$ has a converging subsequence. 
Proof. Since $G$ is bounded on bounded subsets of $\mathscr{M}$, the assumption implies that also

$$
\begin{aligned}
& -D E_{\alpha, \beta}\left(x_{n}, y_{n}\right)\left[G\left(x_{n}, y_{n}\right)\right] \\
= & \left(\left\|\nabla E_{\alpha, \beta}\right\|^{2}+\chi\left(c_{0}-E_{\alpha, \beta+\lambda}\right) \frac{\left\|\nabla E_{\alpha, \beta}\right\|}{\left\|\nabla E_{\alpha, \beta+\lambda}\right\|}\left\langle\nabla E_{\alpha, \beta}, \nabla E_{\alpha, \beta+\lambda}\right\rangle\right)\left(x_{n}, y_{n}\right)
\end{aligned}
$$

is infinitesimal, so Lemma 5.3 implies that there exists $\theta_{n} \in[0,1]$ such that

$$
\left\|\nabla E_{\alpha, \beta+\theta_{n} \lambda}\right\|=\left\|\theta_{n} \nabla E_{\alpha, \beta+\lambda}+\left(1-\theta_{n}\right) \nabla E_{\alpha, \beta}\right\|
$$

is infinitesimal. Up to a subsequence, we may assume that $\left(\theta_{n}\right)$ converges to $\theta \in[0,1]$, and

$$
\nabla E_{\alpha, \beta+\theta \lambda}\left(x_{n}, y_{n}\right)=\nabla E_{\alpha, \beta+\theta_{n} \lambda}\left(x_{n}, y_{n}\right)+\left(\theta_{n}-\theta\right) \lambda y_{n}
$$

is also infinitesimal in $W^{1,2}$, because $\left\|y_{n}^{\prime}\right\|_{2}$ is bounded. By Lemma 4.3 the sequence $\left(x_{n}, y_{n}\right)$ is compact.

6. The Morse homology of $\mathbf{E}_{\alpha, \beta}$. By Lemma 5.1 (i) and (ii), $E_{\alpha, \beta}$ is a nondegenerate Lyapunov function for the smooth Morse vector field $F$ on $\mathscr{M}$. The vector field $F$ is bounded and Lipschitz on bounded sets (condition (F1)) by Lemma 5.2 (i). By Lemma 5.1 (iii), $E_{\alpha, \beta+\lambda}$ is a Lyapunov function for $F$ on the set $\mathscr{A}=$ $\left\{E_{\alpha, \beta+\lambda}<c_{0}-1\right\}$, and $F$ has no rest points on the closure of $\mathscr{A}$ (condition (F2)). The functions $E_{\alpha, \beta}$ and $E_{\alpha, \beta+\lambda}$ are clearly bounded on every bounded subset of $\mathscr{M}$ (condition (F3)). By Lemma 4.2 applied to $\alpha_{0}=\alpha_{1}=\alpha$ and $\beta_{0}=\beta_{1}=\beta$, the set $\left\{E_{\alpha, \beta} \leq c\right\} \cap\left\{E_{\alpha, \beta+\lambda} \geq c^{\prime}\right\}$ is bounded, for every $c, c^{\prime} \in \mathbb{R}$ (condition (F4)). By Lemma 5.4, every bounded Palais-Smale sequence for $\left(F, E_{\alpha, \beta}\right)$ has a converging subsequence (condition (F5)). Finally, conditions (F6) and (F7) are proved in Lemma 4.1 and Lemma 5.2 (ii), respectively. Therefore, the Morse homology $H_{*}\left(E_{\alpha, \beta}\right)$ is well defined.

Let $\left(\alpha_{0}, \beta_{0}\right)$ and $\left(\alpha_{1}, \beta_{1}\right)$ be two elements of $\Gamma\left(s_{0}, \underline{\alpha}, \bar{\alpha}, \beta, \bar{\beta}, a, b\right)$ such that the corresponding energy functionals have only non-degenerate critical points. If

$$
\max \left\{\left\|\alpha_{0}-\beta_{0}\right\|_{\infty},\left\|\alpha_{1}-\beta_{1}\right\|_{\infty}\right\}<\frac{\lambda \underline{\alpha}}{\underline{\alpha}+\bar{\beta}},
$$

Lemma 4.2 implies that the pair $\left(E_{\alpha_{0}, \beta_{0}}, E_{\alpha_{1}, \beta_{1}+\lambda}\right)$ and the pair $\left(E_{\alpha_{1}, \beta_{1}}, E_{\alpha_{0}, \beta_{0}+\lambda}\right)$ satisfy (F8), so the homomorphisms

$$
\Phi_{0,1}: H_{k}\left(E_{\alpha_{0}, \beta_{0}}\right) \rightarrow H_{k}\left(E_{\alpha_{1}, \beta_{1}}\right), \quad \Phi_{1,0}: H_{k}\left(E_{\alpha_{1}, \beta_{1}}\right) \rightarrow H_{k}\left(E_{\alpha_{0}, \beta_{0}}\right),
$$

are well-defined. If

$$
\max \left\{\left\|\alpha_{0}-\beta_{0}\right\|_{\infty},\left\|\alpha_{1}-\beta_{1}\right\|_{\infty}\right\}<\frac{\lambda \underline{\alpha}}{2 \underline{\alpha}+2 \bar{\beta}},
$$

Lemma 4.2 implies that the set

$$
\left\{E_{\alpha_{0}, \beta_{0}} \leq c\right\} \cap\left\{E_{\alpha_{1}, \beta_{1}}+E_{\alpha_{1}, \beta_{1}-\lambda} \geq c^{\prime}\right\}=\left\{E_{\alpha_{0}, \beta_{0}} \leq c\right\} \cap\left\{E_{\alpha_{1}, \beta_{1}-\lambda / 2} \geq c^{\prime} / 2\right\}
$$

is bounded. Since $\left(\alpha_{0}, \beta_{0}+\lambda / 2\right)$ and $\left(\alpha_{1}, \beta_{1}+\lambda\right)$ belong to $\Gamma\left(s_{0}, \underline{\alpha}, \bar{\alpha}, \underline{\beta}+\lambda / 2, \bar{\beta}+\right.$ $\lambda, a, b)$, if

$$
\max \left\{\left\|\alpha_{0}-\beta_{0}\right\|_{\infty},\left\|\alpha_{1}-\beta_{1}\right\|_{\infty}\right\}<\frac{\lambda \underline{\alpha}}{2 \underline{\alpha}+2 \bar{\beta}+\lambda}
$$


Lemma 4.2 implies that the set

$$
\left\{E_{\alpha_{1}, \beta_{1}}+E_{\alpha_{1}, \beta_{1}+\lambda} \leq c\right\} \cap\left\{E_{\alpha_{0}, \beta_{0}+\lambda} \geq c^{\prime}\right\}=\left\{E_{\alpha_{1}, \beta_{1}+\lambda / 2} \leq c / 2\right\} \cap\left\{E_{\alpha_{0}, \beta_{0}+\lambda} \geq c^{\prime}\right\}
$$

is bounded. Therefore, if $\left(\alpha_{0}, \beta_{0}\right)$ and $\left(\alpha_{1}, \beta_{1}\right)$ are so close in the $C^{0}$ norm that $(24)$ holds - and a fortiori also (22) and (23) hold - condition (F9) is satisfied. We conclude that the composition

$$
\Phi_{1,0} \circ \Phi_{0,1}: H_{k}\left(E_{\alpha_{0}, \beta_{0}}\right) \rightarrow H_{k}\left(E_{\alpha_{0}, \beta_{0}}\right)
$$

is the identity. Exchanging the role of $\left(\alpha_{0}, \beta_{0}\right)$ and $\left(\alpha_{1}, \beta_{1}\right)$, we deduce that also the composition

$$
\Phi_{0,1} \circ \Phi_{1,0}: H_{k}\left(E_{\alpha_{1}, \beta_{1}}\right) \rightarrow H_{k}\left(E_{\alpha_{1}, \beta_{1}}\right)
$$

is the identity. We conclude that under assumption (24), the Morse homology of $E_{\alpha_{0}, \beta_{0}}$ is isomorphic to the Morse homology of $E_{\alpha_{1}, \beta_{1}}$.

The set $\Gamma\left(s_{0}, \underline{\alpha}, \bar{\alpha}, \beta, \bar{\beta}, a, b\right)$ is bounded in $C^{0}$, and it contains the product Lorentzian structure $(\underline{\alpha}, \underline{\beta})$. The set of Lorentzian structures $(\alpha, \beta)$ in $\Gamma\left(s_{0}, \underline{\alpha}, \bar{\alpha}, \beta, \bar{\beta}, a, b\right)$ such that $E_{\alpha, \beta}$ is a Morse function is $C^{0}$-dense (actually, $C^{\infty}$ dense). For instance, this follows from the fact that given a globally hyperbolic Lorentzian manifold $(M, h)$ and a point $z_{0} \in M$, the set of points $z_{1} \in M$ which are non-conjugate to $z_{0}$ along every geodesic is residual ${ }^{7}$ (see [Uhl75], Theorem 1 (a)). These facts imply that the Morse homology of every Morse functional $E_{\alpha, \beta}$, $(\alpha, \beta) \in \Gamma\left(s_{0}, \underline{\alpha}, \bar{\alpha}, \underline{\beta}, \bar{\beta}, a, b\right)$, is isomorphic to the Morse homology of $E_{\underline{\alpha}, \underline{\beta}}$ (which we may assume to be a Morse functional on $\mathscr{M}$, by perturbing the metric $g$ ).

The Morse homology of $E_{\underline{\alpha}, \underline{\beta}}$ is isomorphic to the singular homology of $\mathscr{X}$ (see Remark 1.1). The conclusion follows from the fact that $\mathscr{X}$ is homotopically equivalent to $\Omega(X)$, the based loop space of $X$.

\section{REFERENCES}

[ABFm03] A. Abbondandolo, V. Benci, D. Fortunato, and A Masiello, On the Morse inequalities for geodesics on Lorentzian manifolds, Math. Res. Lett. 10 (2003), pp. $435-445$.

[Am01] A. Abbondandolo and P. Majer, Morse homology on Hilbert spaces, Comm. Pure Appl. Math., 54 (2001), pp. 689-760.

[Am05] A. AbBondandolo And P. Majer, A Morse complex for infinite dimensional manifolds - Part I, Adv. Math., 197 (2005), pp. 321-410.

[AM06] A. Abbondandolo and P. Majer, Lectures on the Morse complex for infinite dimensional manifolds, Morse theoretic methods in nonlinear analysis and in symplectic topology (Montreal) (P. Biran, O. Cornea, and F. Lalonde, eds.), Springer, 2006, pp. $1-74$.

[Am08] A. Abbondandolo And P. Majer, A Morse complex for infinite dimensional manifolds - Part II, in preparation, 2008.

[BEe96] J. K. Beem, P. E. Ehrlich, And K. L. Easley, Global Lorentzian geometry, Marcel Dekker, inc., New York, 1996.

[BF94] V. Benci And D. Fortunato, On the existence of infinitely many geodesics on spacetime manifolds, Adv. Math., 105 (1994), pp. 1-25.

\footnotetext{
${ }^{7}$ Indeed, by the above fact we can find a sequence of diffeomorphisms $\left(\varphi_{n}\right)$ of $X \times \mathbb{R}$ of the form $(x, y) \mapsto\left(\varphi_{n}^{1}(x), \varphi_{n}^{2}(y)\right)$ which converge to the identity in the $C^{\infty}$ topology, and such that $\varphi_{n}\left(z_{0}\right)$ and $\varphi_{n}\left(z_{1}\right)$ are non-conjugate along every geodesic. Pulling back the Lorentzian metric by this sequence of diffeomorphisms, we obtain a sequence of globally hyperbolic Lorentzian metrics $C^{\infty}$-converging to the the original one, and for which $z_{0}$ and $z_{1}$ are non-conjugate along every geodesic.
} 
[BFM94] V. Benci, D. Fortunato, And A. Masiello, On the geodesic connectedness of Lorentzian manifolds, Math. Z., 217 (1994), pp. 73-93.

[BS03] A. N. BeRnAl AND M. SÁnChez, On smooth Cauchy hypersurfaces and Geroch's splitting theorem, Commun. Math. Phys., 243 (2003), pp. 461-470.

[BS05] A. N. BERNAL AND M. SÁNCHEZ, Smoothness of time functions and the metric splitting of globally hyperbolic spacetimes, Commun. Math. Phys., 257 (2005), pp. 43-50.

[Flo89] A. FlOER, Witten's complex and infinite-dimensional Morse theory, J. Differential Geom., 30 (1989), pp. 207-221.

[Ger70] R. Geroch, Domain of dependence, J. Mathematical Phys., 11 (1970), pp. 437-449.

[Kli78] W. Klingenberg, Lectures on closed geodesics, Springer, Berlin, 1978.

[Mas94] A. Masiello, Variational methods in Lorentzian geometry, Pitman Research Notes in Mathematics, Longman Scientific \& Technical, Harlow, 1994.

[MPP05] M. Musso, J. Pejsachowicz, and A. Portaluri, A Morse index theorem for perturbed geodesics on semi-Riemannian manifolds, Topol. Methods Nonlinear Anal., 25 (2005), pp. 69-99.

[O'N83] B. O'NeILl, Semi-Riemannian geometry with applications to relativity, Academic Press, New York, 1983.

[Pal63] R. S. Palais, Morse theory on Hilbert manifolds, Topology, 2 (1963), pp. 299-340.

[PP05] P. Piccione AND A. Portaluri, A bifurcation result for semi-Riemannian trajectories of the Lorentz force equation, J. Differential Equations, 210 (2005), pp. 233-262.

[PPT04] P. Piccione, A. Portaluri, And D. Tausk, Spectral flow, Maslov index and bifurcation of semi-Riemannian geodesics, Ann. Global Anal. Geom., 25 (2004), pp. 121149.

[PT02] P. PICCIONE AND D. TAUSk, The Morse index theorem in semi-Riemannian geometry, Topology, 41 (2002), pp. 1123-1159.

[Uhl75] K. UhlenBeCK, A Morse theory for geodesics on a Lorentz manifold, Topology, 14 (1975), pp. 69-90. 
